OPEN ACCESS

Edited by:

Michael Sauer

University of Natural Resources and Life Sciences, Vienna, Austria

Reviewed by:

Vinod Kumar:

Cranfield University, United Kingdom

Mohan Raj Subramanian,

PRIST University, India

${ }^{*}$ Correspondence:

Po-Chi Soo

pcsoo@mail.tcu.edu.tw

†These authors have contributed equally to this work.

Specialty section:

This article was submitted to Microbial Physiology and Metabolism, a section of the journal

Frontiers in Microbiology

Received: 19 September 2017 Accepted: 24 November 2017 Published: 07 December 2017

Citation:

Jeng $W-Y$, Panjaitan NSD, Horng $Y$ - T, Chung W-T, Chien C-C and Soo P-C (2017) The Negative Effects of KPNOO353 on Glycerol Kinase and Microaerobic 1,3-Propanediol Production in Klebsiella pneumoniae. Front. Microbiol. 8:2441. doi: 10.3389/fmicb.2017.02441

\section{The Negative Effects of KPN00353 on Glycerol Kinase and Microaerobic 1,3-Propanediol Production in Klebsiella pneumoniae}

\author{
Wen-Yih Jeng ${ }^{1,2 t}$, Novaria S. D. Panjaitan ${ }^{3 t}$, Yu-Tze Horng ${ }^{4 t}$, Wen-Ting Chung ${ }^{4}$, \\ Chih-Ching Chien ${ }^{5}$ and Po-Chi Soo ${ }^{3,4 *}$
}

\begin{abstract}
${ }^{1}$ University Center for Bioscience and Biotechnology, National Cheng Kung University, Tainan, Taiwan, ${ }^{2}$ Department of Biochemistry and Molecular Biology, National Cheng Kung University, Tainan, Taiwan, ${ }^{3}$ Institute of Medical Sciences, College of Medicine, Tzu Chi University, Hualien, Taiwan, ${ }^{4}$ Department of Laboratory Medicine and Biotechnology, College of Medicine, Tzu Chi University, Hualien, Taiwan, ${ }^{5}$ Graduate School of Biotechnology and Bioengineering, Yuan Ze University, Taoyuan, Taiwan
\end{abstract}

1,3-Propanediol (1,3-PD) is a valuable chemical intermediate in the synthesis of polyesters, polyethers, and polyurethanes, which have applications in various products such as cloth, bottles, films, tarpaulins, canoes, foam seals, high-resilience foam seating, and surface coatings. Klebsiella pneumoniae can produce 1,3-PD from glycerol. In this study, KPN00353, an EllA homologue in the phosphoenolpyruvate (PEP):carbohydrate phosphotransferase system (PTS), was found to play a negative regulatory role in 1,3-PD production under microaerobic conditions via binding to glycerol kinase (GlpK). The primary sequence of KPN00353 is similar to those of the fructose-mannitol ElIA (Ell ${ }^{\mathrm{Fru}}$ and ElIA ${ }^{\mathrm{Mt1}}$ ) family. The interaction between KPN00353 and GlpK resulted in inhibition of the synthesis of glycerol-3-phosphate (G3P) and correlated with reductions in glycerol uptake and the production of 1,3-PD. Based on structure modeling, we conclude that residue $\mathrm{H} 65$ of KPN00353 plays an important role in the interaction with GlpK. We mutated this histidine residue to aspartate, glutamate, arginine and glutamine to assess the effects of each KPN00353 variant on the interaction with GlpK, on the synthesis of G3P and on the production of 1,3-PD. Our results illuminate the role of KPN00353 in 1,3-PD production by $K$. pneumoniae under microaerobic conditions.

Keywords: 1,3-propanediol, glycerol kinase, Klebsiella pneumoniae, carbohydrate phosphotransferase system, EIIA

\section{INTRODUCTION}

Glycerol is a structural component of many lipids (glycerolipids) in organisms, and yeast can produce glycerol by the fermentation of sugar. Since ample glycerol occurs in nature, many microorganisms can utilize glycerol as a sole carbon and energy source (Wang et al., 2001). Klebsiella pneumoniae is not only an opportunistic pathogen but also a saprophytic microorganism that can be found in sewage, soil, plants, insects, animals and humans (Bagley, 1985; Medrano et al., 2014). Glycerol dissimilation in K. pneumoniae can be divided into aerobic metabolism (respiratory metabolism) and two branches of glycerol fermentation. In aerobic metabolism, the dissimilation 
of glycerol is begun by glycerol kinase (GlpK), which synthesizes glycerol-3-phosphate (G3P), followed by oxidation to dihydroxyacetone phosphate (DHAP). Glycerol fermentation by Klebsiella pneumoniae involves two parallel pathways: the reductive and oxidative pathways. The oxidative pathway leads to the production of DHAP. DHAP from either respiratory metabolism or oxidative fermentation is channeled to produce pyruvate. The reductive pathway leads to 1,3-propanediol (1,3-PD) production (Celinska, 2012; Kumar and Park, 2017). The 1,3-PD is a valuable chemical intermediate to produce polyesters, polyethers, polyurethanes and polytrimethylene terephthalate (PTT) (Zeng and Biebl, 2002). 1,3-PD can be produced through either chemical synthesis or microbial conversion. The chemical synthesis processes produce toxic byproducts and require a reduction step under high hydrogen pressure (Sullivan, 1993). In contrast, biosynthesis is a comparatively attractive option because it can use renewable feedstock and does not generate a toxic intermediate. 1,3-PD is a product of glycerol fermentation by Klebsiella, Enterobacter, Citrobacter, Lactobacillus, and Clostridium species and by engineered Escherichia coli (Huang et al., 2002; Saxena et al., 2009; Pyne et al., 2016). Among these organisms, Clostridium butyricum and Klebsiella pneumoniae are considered the best natural 1,3-PD producers (Saxena et al., 2009). However, K. pneumoniae attracts substantial attention because of the availability of genetic engineering tools that are applicable to K. pneumoniae but not C. butyricum (Celinska, 2012; Kumar and Park, 2017). K. pneumoniae can produce 1,3-PD from glycerol under anaerobic, aerobic or microaerobic conditions (Forage and Lin, 1982; Huang et al., 2002; Chen X. et al., 2003; Saxena et al., 2009), but the microaerobic conditions are better than the anaerobic and aerobic conditions (Chen $X$. et al., 2003). In our previous study, the amount of 1,3-PD produced by K. pneumoniae was increased by the overexpression of 1,3-propanediol oxidoreductase (PDOR) or both PDOR and $\mathrm{DhaB}$ under microaerobic conditions (Horng et al., 2010).

The bacterial phosphoenolpyruvate (PEP):carbohydrate phosphotransferase system (PTS) transports and phosphorylates carbohydrates, such as hexoses (e.g., glucose and fructose), sugar alcohols (e.g., mannitol), amino sugars (e.g., $\mathrm{N}$-acetylglucosamine), disaccharides (e.g., cellobiose) and other carbon sources (Al Makishah and Mitchell, 2013; Deutscher et al., 2014; Liang et al., 2015). The PTS is a phosphorylation cascade usually composed of one membrane-spanning protein/domain and four soluble proteins/domains that sequentially transfer phosphate from PEP to the substrate. Enzyme I (EI) and histidine protein $(\mathrm{HPr})$ are the general cytoplasmic PTS proteins involved in the transfer of most PTS carbohydrates in most bacteria. The substrate specificity of the PTS depends on the enzyme II complex, which consists of two soluble proteins/domains (EIIA and EIIB) and a membrane-bound protein/domain (EIIC). In a mannose-type PTS, the enzyme II complex also contains EIID, which is integrated into the membrane. EIIC and EIID facilitate the translocation of the substrate across the cell membrane. Bacteria contain several
EII complexes: for example, $E$. coli contains at least 15 different ones. The phosphoryl transfer chain to phosphorylate the substrate (carbohydrate) begins with EI. PEP is the substrate of EI to provide a phosphoryl group that is then transferred to HPr. The phosphorylated HPr then phosphorylates one of the carbohydrate-specific EIIAs, which in turn passes the phosphoryl group to its cognate, EIIB. Finally, in most PTSs, the phosphorylated EIIB transfers the phosphoryl group to the carbohydrate bound to the cognate EIIC. In addition to sugar transport, the bacterial PTS has been reported to be involved in the regulation of carbohydrate metabolism (including carbohydrate catabolism repression and inducer exclusion), the utilization of a nitrogen source, and potassium uptake via protein-protein interactions. For example, EIIA ${ }^{\text {Glc }}$ (Crr, encoded by $\mathrm{crr}$ and formerly known as IIIglc or IIIGlc) interacts with MalK (the ATP-binding protein of the maltose/maltodextrin ABC transport system) and GlpK in E. coli and Salmonella enterica serovar Typhimurium, and Crr interacts with LacY (lactose permease) in E. coli. These interactions with Crr result in the inducer exclusion-mediated inhibition of MalK, GlpK and LacY and thereby prevent the uptake of maltose, glycerol and lactose, respectively (Deutscher et al., 2006, 2014). Wu et al. reported that in $K$. pneumoniae, the mutation of CelB, a cellobiose-specific EIIC, resulted in impaired biofilm formation (Wu et al., 2012). The peptide sequence identity between Crr in K. pneumoniae and E. coli is $98 \%$. The mutation of $\mathrm{crr}$ in $K$. pneumoniae enhanced 1,3-PD production compared to the parent strain when bacteria were incubated in a mixture of glucose and glycerol under aerobic conditions (Oh et al., 2013).

Guo et al. (2010) reported that the non-capsuled K. pneumoniae provided a higher 1,3-PD yield. In our previous study, we found that the overexpression of KPN00353KPN00352-KPN00351 increased the capsular polysaccharide production in K. pneumoniae. KPN00353 encodes 147amino-acid (aa) putative EIIA homologue. KPN00352 and KPN00351 encode putative EIIB (95 aa) and EIIC (445 aa) homologues respectively (Horng et al., 2017). Therefore, the purpose of this study was to examine the effects of KPN00353-KPN00352-KPN00351 on the 1,3-PD production. This study demonstrates the role of KPN00353, an open reading frame (ORF) common to several K. pneumoniae isolates from different sources, in binding to GlpK, glycerol uptake and 1,3-PD production under microaerobic conditions and provides structural insight into KPN00353-GlpK interaction.

\section{MATERIALS AND METHODS}

\section{Bacterial Strains and Growth Conditions}

The bacterial strains and plasmids used in this study are listed in Table 1. The primers used in this study are listed in Supplementary Table S1. The bacteria were routinely cultured at $37^{\circ} \mathrm{C}$ in Luria-Bertani (LB) medium (10 g/L tryptone, $5 \mathrm{~g} / \mathrm{L}$ yeast extract and $10 \mathrm{~g} / \mathrm{L} \mathrm{NaCl}$ ) supplemented with the appropriate antibiotics. The analysis of 1,3-PD production by 
K. pneumoniae under microaerobic condition was performed by growing the bacteria in $250-\mathrm{mL}$ and sealed flask containing $100 \mathrm{ml}$ minimal culture media (MCM, $4.4 \mathrm{~g} / \mathrm{L} \mathrm{K} \mathrm{HPO}_{4}, 1.3 \mathrm{~g} / \mathrm{L}$ $\mathrm{KH}_{2} \mathrm{PO}_{4}, 2 \mathrm{~g} / \mathrm{L}(\mathrm{NH} 4)_{2} \mathrm{SO}_{4}, 0.2 \mathrm{~g} / \mathrm{L} \mathrm{MgSO}_{4} \cdot 7 \mathrm{H}_{2} \mathrm{O}, 1 \mathrm{~g} / \mathrm{L}$ yeast extract, $20 \mathrm{~g} / \mathrm{L}$ glycerol, $2 \mathrm{~mL} / \mathrm{L}$ trace element solution and $1 \mathrm{~mL} / \mathrm{L} \mathrm{Fe}$ solution, $\mathrm{pH} 7.0$ ) at $37^{\circ} \mathrm{C}$. The trace element solution contained $70 \mathrm{mg} / \mathrm{L} \mathrm{ZnCl}_{2}, 100 \mathrm{mg} / \mathrm{L} \mathrm{MnCl}_{2} \cdot 4 \mathrm{H}_{2} \mathrm{O}$, $60 \mathrm{mg} / \mathrm{L} \mathrm{H}_{3} \mathrm{BO}_{3}, 25 \mathrm{mg} / \mathrm{L} \mathrm{NiCl} \cdot 6 \mathrm{H}_{2} \mathrm{O}, 20 \mathrm{mg} / \mathrm{L} \mathrm{CoCl} \mathrm{Cl}_{2} \cdot 6 \mathrm{H}_{2} \mathrm{O}$, $35 \mathrm{mg} / \mathrm{L} \mathrm{NaMoO} 4 \cdot \mathrm{H}_{2} \mathrm{O}$ and $10.8 \mathrm{mM} \mathrm{HCl}$. The Fe solution contained $5 \mathrm{~g} / \mathrm{L} \mathrm{FeSO}_{4} \cdot 7 \mathrm{H}_{2} \mathrm{O}$ and $48 \mathrm{mM} \mathrm{HCl}$. Microaerobic condition was kept by agitation without aeration (Chen X. et al., 2003).

\section{Reverse Transcription PCR (RT-PCR)}

The bacterial RNA was extracted using the TRI reagent (Sigma-Aldrich, St. Louis, MO, United States). RNA (1.5 $\mu \mathrm{g})$ was reverse transcribed using the Reverse Transcriptase Kit (Qiagen, Hilden, Germany) with random primers. Subsequently,

TABLE 1 | List of strains and plasmids used in the experiments.

\begin{tabular}{|c|c|c|}
\hline Strain & Relevant genotype and phenotype & Reference or source \\
\hline \multicolumn{3}{|l|}{ E. coli strains } \\
\hline $\mathrm{DH} 5 \alpha$ & $\begin{array}{l}\mathrm{F}^{-}, \varphi 80 \mathrm{~d} / a c Z \Delta \mathrm{M} 15,(\operatorname{lac} Z Y A-\arg F) \cup 169, \text { deoR, recA1, endA1, } \\
\text { hsdR17(}\left(\mathrm{rk}^{-}, \mathrm{m}_{k}{ }^{+}\right), \text {phoA, supE44, } \lambda^{-}, \text {thi-1, gyrA96, relA1 }\end{array}$ & Invitrogen \\
\hline S17-1 $\lambda$ pir & $\begin{array}{l}\lambda \text {-pir lysogen of } C C 118[\mathrm{D}(\mathrm{ara}-\mathrm{leu}) \text { araD DlacX } 74 \text { galE galK phoA20 } \\
\text { thi-1 rpsE rpoB argE(Am) recA1]; permissive host for suicide } \\
\text { plasmids requiring the Pir protein }\end{array}$ & Soo et al., 2005 \\
\hline BL21(DE3)pLysS & $\mathrm{F}^{-}$, ompT, gal, dcm, lon, $h s d S_{B}\left(\mathrm{r}_{B}^{-} \mathrm{m}_{B}^{-}\right), \lambda(\mathrm{DE} 3), \operatorname{pLysS}\left(\mathrm{cm}^{R}\right)$ & Novagen \\
\hline \multicolumn{3}{|l|}{ K. pneumoniae strains } \\
\hline MGH78578 & ATCC 700721 & McClelland et al., 2000 \\
\hline KO353 & MGH78578_KPN00353::Sm, Sm ${ }^{r}$ & This work \\
\hline \multicolumn{3}{|l|}{ Plasmid } \\
\hline pGEM-T easy & TA cloning vector, $\mathrm{Ap}^{r}$ & $\begin{array}{l}\text { Promega, Fitchburg, WI, } \\
\text { United States }\end{array}$ \\
\hline pGEX-1-Sall & $\begin{array}{l}\text { GST tag expression vector constructed from pGEX-1 by inserting } \\
\text { the restriction enzyme site Sall between ECORI and Smal. }\end{array}$ & This work \\
\hline pGEX-1::glpK::km & Expressing GST-tagged GlpK, $\mathrm{Km}^{r}$ & This work \\
\hline pET30b & Expression vector, His-tag, $\mathrm{Km}^{r}$ & $\begin{array}{l}\text { Novagen (merged with } \\
\text { Merck-Millipore, Darmstadt, } \\
\text { Germany) }\end{array}$ \\
\hline pET30b::353WT & Expressing His-tagged KPN00353, $\mathrm{Km}^{r}$ & This work \\
\hline pET30b::H65Q & Expressing His-tagged mutated KPN00353(H65Q), Km & This work \\
\hline pET30b::H65D & Expressing His-tagged mutated KPN00353(H65D), $\mathrm{Km}^{r}$ & This work \\
\hline pET30b::H65E & Expressing His-tagged mutated KPN00353(H65E), $\mathrm{Km}^{r}$ & This work \\
\hline pET30b::H65R & Expressing His-tagged mutated KPN00353(H65R), $\mathrm{Km}^{r}$ & This work \\
\hline pET30b::H110Q & Expressing His-tagged mutated KPN00353(H110Q), Km & This work \\
\hline pET30b::mrkD & Expressing His-tagged MrkD, $\mathrm{Km}^{r}$ & This work \\
\hline pET30a::KPN00350 & Expressing His-tagged KPN00350, $\mathrm{Km}^{r}$ & This work \\
\hline pET21::crr & Expressing His-tagged Crr (KPN02764), Amp ${ }^{r}$ & This work \\
\hline pBlueScript II SK+ (pBSK) & pBR322 ori, lac promoter, Amp ${ }^{r}$ & $\begin{array}{l}\text { Stratagene (La Jolla, CA, } \\
\text { United States) }\end{array}$ \\
\hline pBSK-Gm & $\begin{array}{l}\text { Gentamycin resistance gene was inserted into the Scal site within } \\
\text { the ampicillin resistance gene in } \mathrm{pBSK}, \mathrm{Gm}^{r}\end{array}$ & This work \\
\hline pBSK-51-53-GM & $\begin{array}{l}\text { pBSK::Gm containing KPN00353-KPN00352-KPN00351 located } \\
\text { downstream of lac promoter, } \mathrm{Gm}^{r}\end{array}$ & This work \\
\hline pBSK-52-53-GM & $\begin{array}{l}\text { pBSK::Gm containing KPN00353-KPN00352) located downstream } \\
\text { of lac promoter, } \mathrm{Gm}^{r}\end{array}$ & This work \\
\hline pBSK-53-GM & $\begin{array}{l}\text { pBSK::Gm containing KPN00353 located downstream of lac } \\
\text { promoter, Gm }{ }^{r}\end{array}$ & This work \\
\hline pBAD33 & $\mathrm{P}_{\mathrm{BAD}}$ promoter, pACYC184 ori, $\mathrm{Cm}^{r}$ & Guzman et al., 1995 \\
\hline pBAD33::Histaq353WT & pBAD33 containing KPN00353, $\mathrm{Cm}^{r}$ & This work \\
\hline pBAD33::Histaq353H65Q & pBAD33 containing mutated KPN00353(H65Q), $\mathrm{Cm}^{r}$ & This work \\
\hline pBAD33::Histaq353H65D & pBAD33 containing mutated KPN00353(H65D), $\mathrm{Cm}^{r}$ & This work \\
\hline pBAD33::Histaq353H65E & pBAD33 containing mutated KPN00353(H65E), $\mathrm{Cm}^{r}$ & This work \\
\hline pBAD33::Histaq353H65R & pBAD33 containing mutated KPN00353(H65R), $\mathrm{Cm}^{r}$ & This work \\
\hline pBAD33::Histaq353H110Q & pBAD33 containing mutated KPN00353 (H110Q), Cm & This work \\
\hline
\end{tabular}


the cDNA of each junction of KPN00353-KPN00348 was amplified by PCR using cDNA $(1 \mu \mathrm{L})$, primer pairs $(400 \mathrm{nM}$; Figure 2 and Supplementary Table S1), PCR buffer, dNTPs, and Taq polymerase according to the manufacturer's instructions (Takara, Japan). The PCR conditions were $1 \mathrm{~min}$ at $95^{\circ} \mathrm{C}$ followed by 32 cycles of $15 \mathrm{~s}$ at $94^{\circ} \mathrm{C}, 30 \mathrm{~s}$ at $60^{\circ} \mathrm{C}$, and $30 \mathrm{~s}$ at $72^{\circ} \mathrm{C}$.

\section{Construction of K. pneumoniae Mutant Strain K0353}

Genetic methods based on homologous recombination were used to amplify the central region of the KPN00353 gene by PCR using the primer pair 353F/353R (Supplementary Table S1). The PCR products were TA-cloned into pGEM-T vectors (Table 1), excised as EcoRI fragments, and ligated with streptomycin-resistant $\Omega$ cassettes into tnp-deleted pUT vectors to form suicide plasmids (Soo et al., 2005). The suicide plasmids containing the central DNA region of KPN00353 were transferred to K. pneumoniae MGH 78578 by electroporation. The transformants were spread on LB plates containing streptomycin $(50 \mu \mathrm{g} / \mathrm{mL})$. Mutant candidates were screened by colony PCR. PCR or Southern blot hybridization was performed to confirm the mutant genotypes.

\section{Analysis of 1,3-PD and Glycerol by High-Performance Liquid Chromatography (HPLC)}

To quantify the 1,3-PD of K. pneumoniae MGH 78578, KO353 or bacteria containing the recombinant DNA under the control of the lac promoter, pre-cultures were grown overnight at $37^{\circ} \mathrm{C}$ in LB medium containing the appropriate antibiotics. Subsequently, the pre-cultures were diluted 100-fold and grown in MCM (supplemented with isopropyl $\beta$-D-1-thiogalactopyranoside (IPTG) at $1 \mathrm{mM}$ for the bacteria containing the lac promoter) under microaerobic conditions for an additional $12 \mathrm{~h}$ at $37^{\circ} \mathrm{C}$ in a shaking incubator at $200 \mathrm{rpm}$ without aeration before collection of the bacterial culture supernatant and quantification of 1,3-PD. To quantify the 1,3-PD in bacteria containing the recombinant DNA under the control of $\mathrm{P}_{\mathrm{BAD}}$, the pre-cultures were diluted 100 -fold in $\mathrm{LB}$ containing the appropriate antibiotics and grown for $2 \mathrm{~h}$ at $37^{\circ} \mathrm{C}$ under microaerobic conditions in a shaking incubator at $200 \mathrm{rpm}$ without aeration. Then, arabinose $(0.4 \%)$ was added to the medium for an additional $3 \mathrm{~h}$ at $30^{\circ} \mathrm{C}$ in a shaking incubator at $150 \mathrm{rpm}$. Then, the bacteria were changed to fresh MCM for an additional $4 \mathrm{~h}$ at $37^{\circ} \mathrm{C}$ before collection of the bacterial culture supernatant and quantification of 1,3-PD.

The bacterial growth was monitored by measuring the optical density of the broth culture at $600 \mathrm{~nm}$, and then the bacterial culture supernatant was collected by centrifugation at $16,200 \times g$ for $2 \mathrm{~min}$ and filtered using a nylon syringe-driven filter (Advangene, Lake Bluff, IL, United States). The concentrations of glycerol and 1,3-PD in the culture supernatant were determined using an HPLC system (Hitachi, Japan) equipped with an ICsep COREGEL-87H3 column (Transgenomic, San Jose, CA, United States) and a refractive index detector (L-2490). The column temperature was set to $65^{\circ} \mathrm{C}$, and $5 \mathrm{mM} \mathrm{H}_{2} \mathrm{SO}_{4}$ was used as the mobile phase with a flow rate of $0.4 \mathrm{~mL} / \mathrm{min}$. The specific 1,3-PD production was defined as the amount of 1,3-PD per $\mathrm{OD}_{600}$ value of bacterial density.

\section{Construction of the Recombinant Plasmids pGEX-1::glpk::km and pET30b::353WT}

Full-length $g l p K(K P N 04011)$ and KPN00353 were amplified by PCR using the chromosomal DNA of K. pneumoniae MGH 78578 and the primer pairs pGEX-glpK-FP/pGEX-glpK-RP and pET30b_353_FP/pET30b_353_RP, respectively (Supplementary Table S1). The PCR products of $g l p K$ and KPN00353 were cloned into the pGEM-T easy vector (Promega, Fitchburg, WI, United States). Subsequently, the $g l p K$ fragment and the gene coding for kanamycin resistance were simultaneously cloned into the SmaI/SalI sites of pGEX-1-SalI (Table 1) to generate the pGEX-1::glpK::km plasmid for expressing glutathione S-transferase (GST)-tagged GlpK (KpGlpK). The $K P N 00353$ DNA fragment was subcloned from the pGEM-T vector containing KPN00353 into the SalI site of the pET30b vector (Novagen merged with Merck-Millipore Darmstadt, Germany) to generate the pET30b::353WT plasmid for expressing His-tagged KPN00353. The construction of the plasmids pET30b::mrkD, pET30a::KPN00350 and pET21::crr followed the same method used to construct pET30b::353WT with the appropriate primer pairs for the respective clones (Supplementary Table S1).

\section{Site-Directed Mutagenesis of KPN00353}

The KPN00353 variants in pET30b::H65Q, pET30b::H65D, pET30b::H65E, pET30b::H65R and pET30b::H110Q were constructed by using the QuikChange ${ }^{\circledR} \quad$ Site-Directed Mutagenesis Kit (Stratagene, La Jolla, CA, United States) according to the manufacturer's instructions. The primers are listed in Supplementary Table S1. The PCR conditions were as follows: $95^{\circ} \mathrm{C}$ for $5 \mathrm{~min}$ followed by 30 cycles of $95^{\circ} \mathrm{C}$ for $1 \mathrm{~min}, 50^{\circ} \mathrm{C}$ for $30 \mathrm{~s}$, and $72^{\circ} \mathrm{C}$ for $6 \mathrm{~min}$. The final step was an additional $72^{\circ} \mathrm{C}$ for $6 \mathrm{~min}$. The template was pET30b::353WT (Table 1). The KPN00353 variants in pBAD33::Histaq353H65Q, pBAD33::Histaq353H65D, pBAD33::Histaq353H65E, pBAD33:: Histaq353H65R, and pBAD33::Histaq353H110Q were cloned from pET30b::H65Q, pET30b::H65D, pET30b::H65E, pET30b::H65R, and pET30b::H110Q, respectively, by XbaI and HindIII digestion followed by insertion into pBAD33 (Guzman et al., 1995).

\section{Protein Pull-Down Assay}

The GST fusion and His-tagged fusion proteins were produced by culturing E. coli DH5 $\alpha$ containing pGEX-1::glpK::km and E. coli BL21(DE3)pLysS containing pET30b::353WT (or pET30b carrying a His-tagged KPN00353 point-mutated gene), respectively, in $5 \mathrm{~mL}$ of $\mathrm{LB}$ medium containing antibiotics overnight. One hundred microliters of the overnight culture was transferred to $10 \mathrm{~mL}$ of LB broth containing antibiotics. After incubation at $37^{\circ} \mathrm{C}$ and $220 \mathrm{rpm}$ for $2 \mathrm{~h}$, IPTG was added at a 
final concentration of $0.5 \mathrm{mM}$ for induction. After an additional $3 \mathrm{~h}$ of culture, the bacterial cells were pelleted by centrifugation $(4000 \times g$ for $10 \mathrm{~min})$ and then suspended in $2 \mathrm{~mL}$ of STE buffer (10 mM Tris- $\mathrm{mCl}, \mathrm{pH} 8.0,150 \mathrm{mM} \mathrm{NaCl}, 1 \mathrm{mM}$ EDTA, $1 \%$ Triton X-100, $1 \mathrm{mM}$ PMSF, $1 \mu \mathrm{g} / \mathrm{mL}$ pepstatin, $1 \mu \mathrm{g} / \mathrm{mL}$ leupeptin and $1 \mathrm{mM}$ DTT). The bacterial cells were frozen at $-70^{\circ} \mathrm{C}$ overnight, lysed by sonication, and then centrifuged $\left(4^{\circ} \mathrm{C}\right.$, $16,200 \times g, 10 \mathrm{~min})$. Two hundred microliters of supernatant containing GST-KpGlpK and $800 \mu \mathrm{L}$ of supernatant containing His-tagged proteins were mixed and incubated with mild shaking at $4^{\circ} \mathrm{C}$ for $1 \mathrm{~h}$ to allow the proteins to interact. Glutathionesepharose (GSH) beads ( $50 \mu \mathrm{L}$; GE Healthcare, United Kingdom) were then added to the spent supernatant, and the mixture was incubated with mild shaking for $1 \mathrm{~h}$ at $4^{\circ} \mathrm{C}$. After the beads were washed three times with $\mathrm{PBS}(\mathrm{pH} 7.4)$, the proteins were separated by $12.5 \%$ sodium dodecyl sulfate-polyacrylamide gel electrophoresis (SDS-PAGE) and detected by Western blotting using anti-His antibody or anti-GST antibody.

\section{Western Blot Analysis}

The Western blot procedures were modified from those described by Sambrook et al. (1989). The proteins were analyzed by $12.5 \%$ SDS-PAGE. The separated proteins were transferred to a nitrocellulose membrane (Pall, Washington, NY, United States) and incubated in blocking buffer (PBS with 5\% milk and $0.1 \%$ Tween 20) for $1 \mathrm{~h}$. Further incubation with anti-His monoclonal antibody (1:2500, Invitrogen) was performed in blocking buffer for $1 \mathrm{~h}$ at room temperature, and the membranes were then washed three times with PBS containing $0.1 \%$ Tween 20. After horseradish peroxidase-conjugated anti-mouse IgG antibody (1:2500, Invitrogen) was added, the membranes were incubated for $1 \mathrm{~h}$, washed three times with PBS containing $0.1 \%$ Tween 20, and reacted with ECL Plus Solution (GE Healthcare, United Kingdom) for $1 \mathrm{~min}$. The intensities of the bands were detected using the Gel Catcher 2850 chemiluminescence camera system (CLUBIO, Taipei, Taiwan).

\section{Quantification of Intracellular Glycerol 3-Phosphate (G3P)}

Klebsiella pneumoniae containing pBAD33, pBAD33::Histaq KPN00353, pBAD33::Histaq353H65Q, pBAD33::Histaq 353H65D, pBAD33::Histaq353H65E, pBAD33::Histaq353H65R, or pBAD33::Histaq353H110Q was grown overnight at $37^{\circ} \mathrm{C}$ in LB medium containing chloramphenicol $(100 \mu \mathrm{g} / \mathrm{mL})$. Then, the pre-cultures were diluted 100-fold in fresh LB medium containing chloramphenicol $(100 \mu \mathrm{g} / \mathrm{mL})$. After the cultures were grown at $37^{\circ} \mathrm{C}$ in a shaking incubator at $200 \mathrm{rpm}$ for $2 \mathrm{~h}$, $0.4 \%$ arabinose was added for protein induction. After induction at $30^{\circ} \mathrm{C}$ and $150 \mathrm{rpm}$ for $3 \mathrm{~h}$, the bacteria were centrifuged and resuspended in fresh MCM. After 4 h of growth, the cell density was measured by spectrophotometry at $600 \mathrm{~nm}$. An aliquot $[\mathrm{OD} \times \mathrm{V}(\mathrm{ml})=1]$ of cells was centrifuged and homogenized in $400 \mu \mathrm{L}$ of G3P assay buffer provided in the Glycerol 3Phosphate Colorimetric Assay Kit (Sigma-Aldrich, St Louis, MO, United States). The intracellular G3P was then measured according to the manufacturer's instructions using the Glycerol
3-Phosphate Colorimetric Assay Kit (Sigma-Aldrich, St Louis, MO, United States).

\section{Structural Modeling}

To predict the interface residues of the $K$. pneumoniae KpGlpK-KPN00353 complex from a structural perspective, 3D structural models of K. pneumoniae GlpK (KpGlpK) and KPN00353 were created using the HHpred server (Soding et al., 2005), a website that uses HMM-HMM comparisons to detect homology and predict structures. The structural model of $K p \mathrm{GlpK}$ was generated using the top 10 templates with the highest scores (PDB accession codes: 3H3N_X, 3G25_A, 3EZW_A, 2D4W_A, 2DPN_A, 2ZF5_O, 2W40_A, 4ELJ_A, 3LL3_A, and 3WXL_A). The structural model of KPN00353 was generated using seven templates selected from the nine templates with highest scores (PDB accession codes: 3OXP_A, 1A3A_A, 1A6J_A, 2A0J_A, 3BJV_A, 3URR_A, and 2OQT_A). The structural model of K. pneumoniae KpGlpK-KPN00353 complex was generated using the ZDOCK server (Chen R. et al., 2003), a website that performs a full rigid-body search of docking orientations between two proteins. The top docking model of the KpGlpK-KPN00353 complex was superimposed with the E. coli GlpK-Crr complex structure (PDB accession code: 1GLA) using the PDBeFold server (Krissinel and Henrick, 2004) to produce structural figures. The models of the KPN00353 mutants were generated by the Coot software (Emsley and Cowtan, 2004). The interface residues of all complex structures were determined by PDBePISA (Krissinel and Henrick, 2007), a website that provides an interactive tool for exploring macromolecular interfaces. Structural figures were then produced using PyMOL (DeLano Scientific ${ }^{1}$ ).

\section{Statistical Methods}

For all quantitative data, the values were expressed as mean \pm standard deviation from three independent bacterial cultures. Paired Student's $t$-test was performed to determine statistically significant differences, and $p<0.05$ was considered to indicate statistical significance. For RT-PCR, Western blotting and SDS-PAGE, the representative data were chosen from three independent experiments.

\section{RESULTS}

\section{The Locus of KPNOO353 and Its Downstream Region Is Common to Most K. pneumoniae Isolates}

To see whether the ORFs KPN00353-KPN00352-KPN00351 are specific to $K$. pneumoniae MGH 78578 or common to most K. pneumoniae isolates, we randomly selected eight $K$. pneumoniae strains from the NCBI database and compared their genomes with that of $K$. pneumoniae MGH 78578. These nine K. pneumoniae isolates were isolated from different sources including sputum, rectal swab, blood, liver abscess, skin, wound

\footnotetext{
${ }^{1}$ http://www.pymol.org
} 

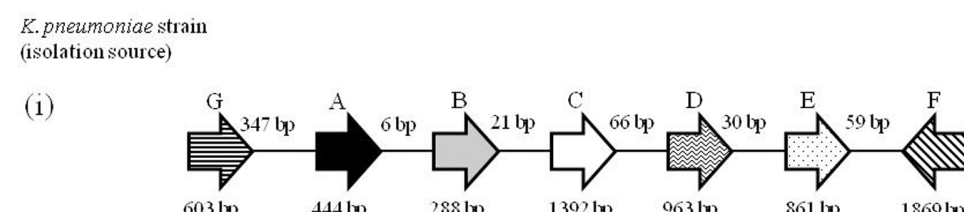

old locus tag of $\mathrm{G}-\mathrm{F}$

(new locus tag of $\mathrm{G}-\mathrm{F}$ )

MGH78578

(sputum)

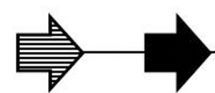

288 bp

1392 bp 963 bp

861 bp $\quad 1869$ bp

KPN 00354-KPN 00348

(KPN_RS01895-KPN_RS01860)

NTUH-K2044
(blood)
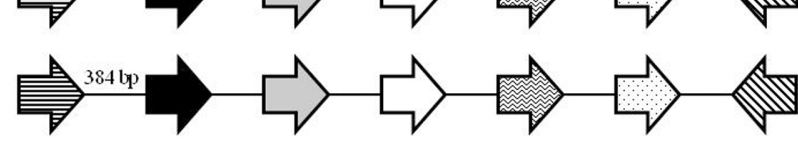

KP1_1218-KP1_1210

TGH13

TGH13
(rectal swab)

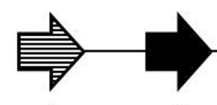

$\Rightarrow-5$

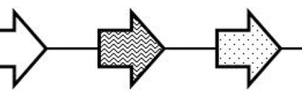

(1)

(KP1_RS05780-KP1_RS05745)

UHKPC33

(hospital; KPC)
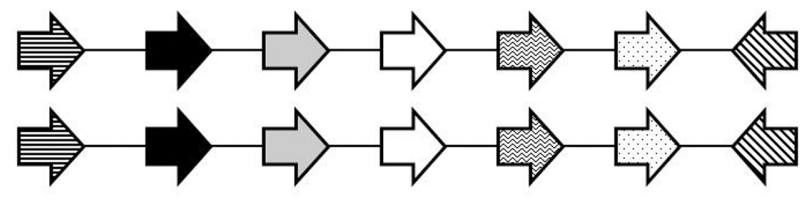

AOG30_21570-AOG30_21600

(AOG30_RS21640-AOG30_RS21670)

KP-5

(hospital;NDM)

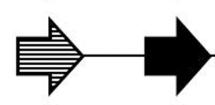

KP-1

(envir onment)
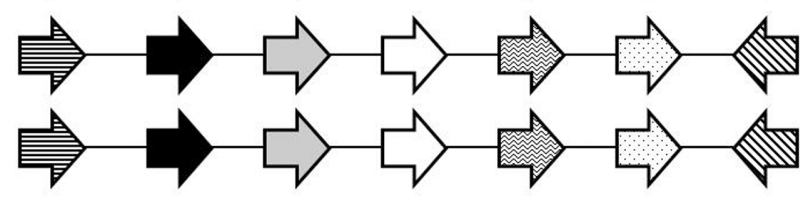

H222_20970-H222_21005

(H222_RS20950-H222_RS20985)

(ii)
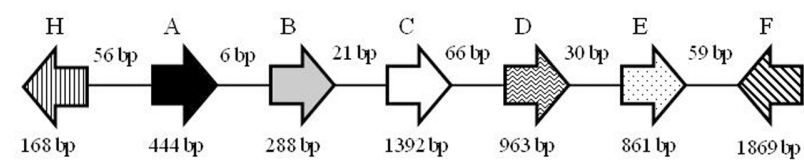

locus tag of H-F

ACG94_07950-ACG94_07980

(ACG94_RS08475-ACG94_RS08505)

KLP1_18515-KLP1_18480

(KLP1_RS18560-KLP1_RS18525)

Geo-822-917
(skin)
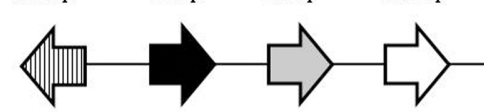

963 tp $\quad 861$ bp $\quad 1869$ lp

loctstag or H-F

MNCRE53
(wound)
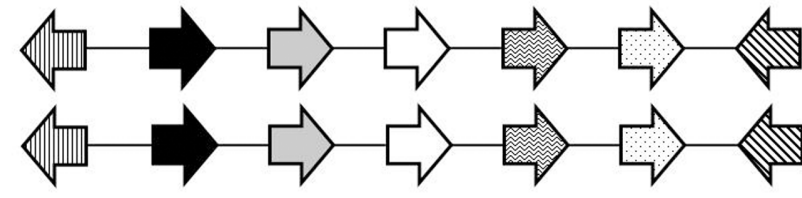

BB746_21395-BB746_21425

CG43

(liver abscess)
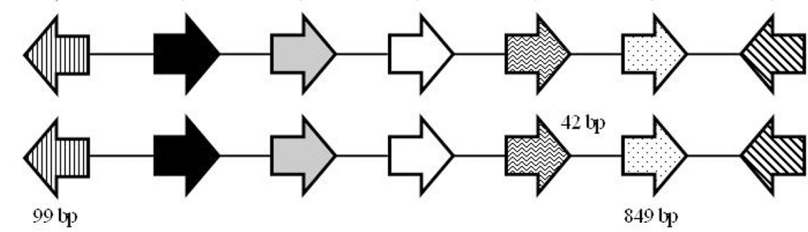

BTE50_07930-BTE50_07900

FIGURE 1 | Alignment of the KPNO0354-KPN00348 locus in different $K$. pneumoniae isolates. Open reading frames (ORFs) A, B, C, D, E, F and G are homologues of KPN00353, KPN00352, KPN00351, KPN00350, KPN00349, KPN00348 and KPN00354, respectively. ORF A is an EllA homologue (black arrow). ORF B is an EIIB homologue (gray arrow). ORF C is an EIIC homologue (white arrow). ORF D is a protein containing the PfkB-specific motif (arrow with wavy line inside). ORF E is a putative type II Fba (arrow with dot inside). ORF F is predicted to encode amidohydrolase (arrow with slash inside). ORF G is predicted to encode peroxiredoxin (arrow with horizontal line). ORF $\mathrm{H}$ is a hypothetical protein (arrow with vertical line). Arrows denote the direction of transcription. The gene lengths and the lengths between each ORF are shown in representative graphs but the lengths different from representative graphs are shown on separate graphs.

and environmental samples. Among them, one NDM-positive strain and one KPC-positive strain were chosen to test whether isolates with these drug-resistant phenotypes have the locus KPN00353-KPN00352-KPN00351 (Figure 1). The results showed that the locus from KPN00353 to its 6-kb downstream region can be observed in all K. pneumoniae isolates. However, these nine isolates can be separated into two groups according to the upstream region of KPN00353. One group consists of isolates with a peroxiredoxin (KPN00354) located 347 bp upstream of KPN00353 in most isolates or $384 \mathrm{bp}$ upstream of the KPN00353 homologue in NTU-K2044. The other group consists of isolates with an ORF of unknown function located 56 bp upstream of the KPN00353 homologue. This 6-kb locus cannot be found in the genome of Klebsiella oxytoca. Therefore, we presume that the observation in this study of KPN00353 in K. pneumoniae MGH 78578 is common to most K. pneumoniae isolates.

The peptide sequence from the 23rd to the 299th residue of KPN00350 contains the PfkB-specific motif. The gene KPN00349 encodes a 286-amino-acid product that is a putative type II Fba. The distances between KPN00353 and KPN00352, KPN00352 and KPN00351, KPN00351 and KPN00350, and KPN00350 and KPN00349 were found to be 5, 74, 65 and 29 bp, respectively. Therefore, we predicted these five genes to be in an operon. To determine the transcriptional unit containing these five genes, reverse transcription PCR (RT-PCR) was performed using the total RNA extracted from K. pneumoniae MGH 78578 and primers that specifically amplify the regions between each pair 

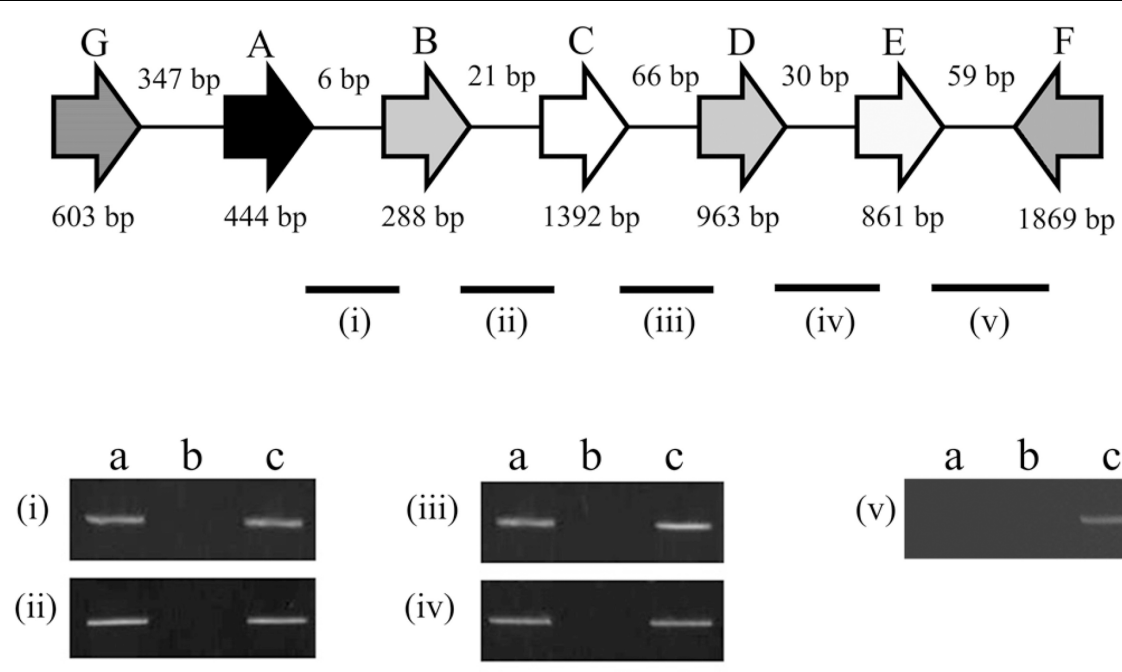

(iii)

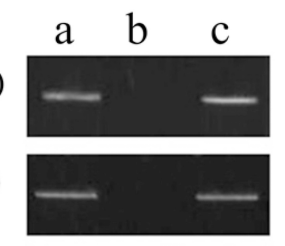

(v)

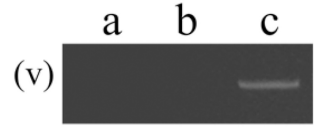

FIGURE 2 | Schematic diagram of the genetic organization of KPN00353 (A), KPN00352 (B), KPN00351 (C), KPN00350 (D), KPN00349 (E), KPN00348 (F) and KPN00354 (G) in K. pneumoniae MGH 78578. Arrows denote the direction of transcription. Each line indicates a junction (i, ii, iii, iv, and v) between the ORFs. The RNA extracted from wild-type K. pneumoniae was analyzed by RT-PCR (a) or PCR (b). The genomic DNA of the wild-type strain was analyzed by PCR as a positive control (c).

of ORFs (junctions i, ii, iii and iv in Figure 2). The transcripts were detected with each primer pair, demonstrating that KPN00353, KPN00352, KPN00351, KPN00350 and KPN00349 are transcribed into a polycistronic mRNA (Figure 2). KPN00348 is located downstream and in the opposite direction of KPN00349. $K P N 00348$ is predicted to encode amidohydrolase (Figures 1, 2). The region between KPN00349 and KPN00348 in the extracted RNA, as a negative control, could not be amplified by RT-PCR using the corresponding primers (junction $\mathrm{V}$ in Figure 2). Therefore, KPN00353, KPN00352, KPN00351, KPN00350 and $K P N 00349$ are in an operon.

\section{Overexpression of KPN00353 Represses 1,3-PD Production in K. pneumoniae}

We observed reduced 1,3-PD production in this study (Figure 3A) and increased capsular polysaccharide production in the previous study (Horng et al., 2017) in K. pneumoniae overexpressing KPN00353-KPN00352-KPN00351 incubated under microaerobic conditions compared with that of the vector control. This finding is consistent with the study by Guo et al. (2010) reporting that non-capsulated K. pneumoniae provided a higher 1,3-PD yield. Then, we tried to detect which ORF(s) of KPN00353-KPN00352-KPN00351 affected the 1,3-PD production by constructing plasmids controlling the expression of KPN00352-KPN00353 or KPN00353 by an inducible lac promoter and then transforming these plasmids separately into K. pneumoniae MGH 78578. The results showed that reductions in the level of 1,3-PD production were similar in bacteria overexpressing KPN00353-KPN00352-KPN00351, KPN00353-KPN00352 or KPN00353 but not in the vector control (Figure 3A). Therefore, we conclude that KPN00353 affects 1,3-PD production in K. pneumoniae. Subsequently, a KPN00353 mutant KO353 was constructed. The 1,3-PD production and growth in the KO353 and wild-type were not significantly different, indicating that the deficiency of KPN00353 did not affect the 1,3-PD production in $K$. pneumoniae (Figures 3A,C). Since 1,3-PD is produced from glycerol fermentation in $K$. pneumoniae, we observed the residual glycerol in the medium after a 12-h bacterial culture under microaerobic conditions. HPLC analysis showed that $9.46 \mathrm{~g} / \mathrm{L}$ glycerol remained in the medium after culturing $K$. pneumoniae overexpressing KPN00353 (Figure 3A). However, 5.82 g/L glycerol remained in the medium after incubating $K$. pneumoniae containing the vector control (Figure 3A). To determine whether the growth was affected by the glycerol uptake, we examined the bacterial growth, and the results showed no significant difference between $K$. pneumoniae containing the vector control or overexpressing KPN00353 (Figure 3B). These results indicated that overexpression of KPN00353 reduced 1,3-PD production and glycerol uptake by K. pneumoniae without affecting bacterial growth in a medium containing glycerol and yeast extract as the carbon sources under microaerobic conditions.

\section{The Interaction of KPN00353 and GIpK Inhibits GlpK Activity}

Iterative database searches performed using the protein BLAST (BLASTP) suite demonstrated that the primary sequence of KPN00353 was similar to that of the fructose-mannitol EIIA (EII $^{\mathrm{Fru}}$ and EIIA ${ }^{\mathrm{Mtl}}$ ) family, a subfamily of the glucose-fructoselactose PTS superfamily (Figure 4). Crr is a glucose-specific EIIA $\left(\right.$ EIIA $^{\mathrm{Glc}}$ ) and is classified as part of the glucose family, another subfamily of the glucose-fructose-lactose PTS superfamily. Unphosphorylated Crr protein in E. coli and S. enterica serovar Typhimurium was reported to interact with GlpK, leading to the inhibition of glycerol uptake (Deutscher et al., 2006, 2014). The primary peptide sequences of Crr and KPN00353 are not similar 


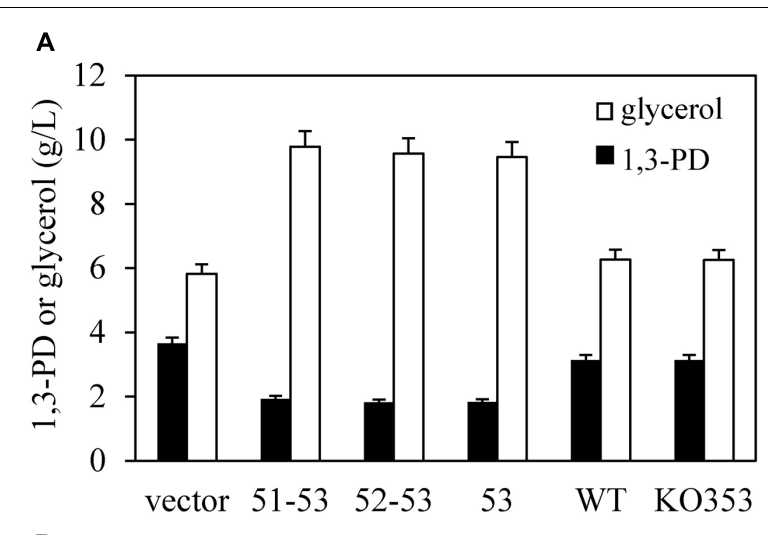

B

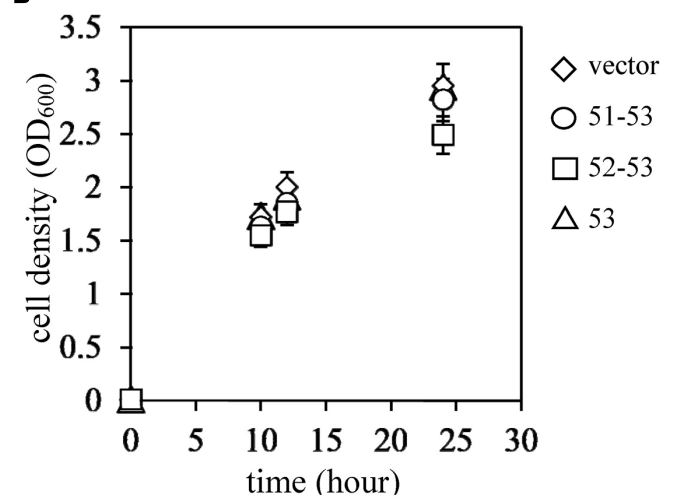

C

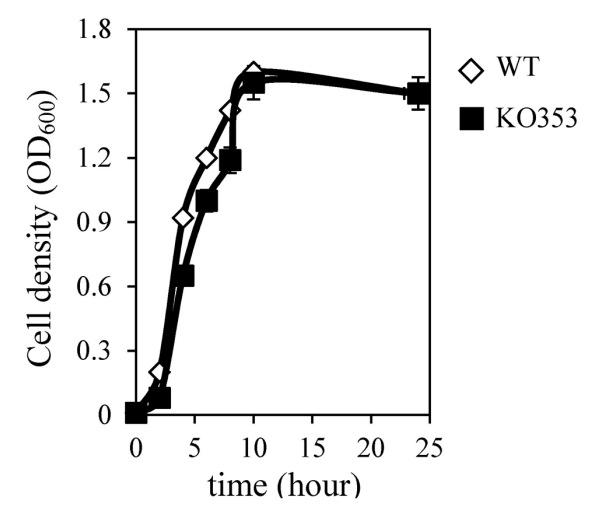

FIGURE 3 | Quantification of 1,3-PD, glycerol and cell density. (A) The amount of 1,3-PD (black bar) and residual glycerol (white bar) in the medium after $12 \mathrm{~h}$ of bacterial culture. (B) The bacterial density was measured at $\mathrm{OD}_{600}$ when the bacteria were inoculated and after growing for 10, 12, and $24 \mathrm{~h}$. vector, K. pneumoniae containing pBSK-Gm as vector control (white diamond). 51-53, K. pneumoniae containing the plasmid pBSK-51-53-GM to overexpress KPN00353, KPN00352 and KPN00351 (white circle). 52-53, K. pneumoniae containing the plasmid pBSK-52-53-GM to overexpress KPN00353 and KPN00352 (white square). 53, K. pneumoniae containing the plasmid pBSK-53-GM to overexpress KPN00353 (white triangle). (C) The bacterial density was measured at $\mathrm{OD}_{600}$. White diamond, wild type (WT). Black square, KPN00353 mutant (KO353).

and are classified into two different subfamilies. However, we still attempted to test whether KPN00353 interacted with KPN04011, the GlpK homologue in K. pneumoniae MGH 78578. KPN04011 was constructed downstream of the GST gene in the plasmid
pGEX-1::glpK::km to generate GST-tagged KpGlpK (Table 1). The plasmid pET30b::KPN00353 was constructed to express His-tagged KPN00353 (Table 1). In addition, His-tagged MrkD, the type 3 fimbrial adhesin (Schurtz et al., 1994), was purified and used as a negative binding control. The GST-KpGlpK, GST and His-tagged proteins were expressed in E. coli. GST-KpGlpK or GST was immobilized on GSH-Sepharose beads. His-tagged KPN00353 was pulled down by GST-KpGlpK but not by GST alone (Figure 5A). Furthermore, His-tagged MrkD was not pulled down by GST-KpGlpK (Figure 5B). These results indicated that KPN00353 interacted specifically with KpGlpK.

Glycerol is phosphorylated to G3P by GlpK (Wehtje et al., 1995). To examine the effect of the binding of KPN00353 and GlpK on the activity of GlpK, we quantified the intracellular G3P in recombinant K. pneumoniae either overexpressing KPN00353 or containing the vector, respectively. Compared to the vector control, K. pneumoniae overexpressing wild-type KPN00353 decreased the intracellular G3P concentration (Figure 6). This decrease indicated that the interaction of KPN00353 and GlpK inhibited the activity of GlpK in K. pneumoniae.

\section{Structural Models of KPN00353 Bound to GlpK of $K$. pneumoniae}

The primary sequence of KPN00353 is similar to that of the fructose-mannitol EIIA (EII ${ }^{\mathrm{Fru}}$ and EIIA ${ }^{\mathrm{Mtl}}$ ) family, according to the sequence analysis by BLASTP. Owing to the absence of structural information on the fructose-mannitol family proteins interacting with GlpK, we generated individual structural models of KpGlpK and KPN00353 using the HHpred server and a complex structural model of $K p \mathrm{GlpK}$ interacting with KPN00353 using the ZDOCK server, which provided a structural perspective for understanding the interactions between $K p \mathrm{GlpK}$ and KPN00353 (Figure 7 and Table 2). Our KpGlpK-KPN00353 complex model revealed that KPN00353 and KpCrr might use individual binding sites to interact with $K p \mathrm{GlpK}$ (Figure 7). Comparing the protein structures of EII ${ }^{\mathrm{Fru}} / \mathrm{EIIA}^{\mathrm{Mtl}}$ to EIIA ${ }^{\mathrm{Glc}}$ and EIIA ${ }^{\text {Lac }}$ showed that the position of His65 in KPN00353 was similar to that of His91 of EcCrr (EIIA ${ }^{\text {Glc }}$ in E. coli) (Figures 7D,E). Comparing the predicted KPN00353 structure and the solved EIIA ${ }^{\text {Fru }}$ structures showed that another key conserved residue in KPN00353 is His110, which is proximal to the His65 of KPN00353 residues (Figure 7E). The structural position of His110 in KPN00353 is similar to that of His76 in EcCrr (Figures 7D,E) (Hurley et al., 1993; Sliz et al., 1997; Bordo et al., 1998). Both key conserved residues involved in the phosphorylation regulation of KPN00353, His65 and His110, are located at the center of the interface of KpGlpK-KPN00353 complex (Figure 7E). The interface residues of the EcGlpK-Crr complex (solved structure) and the KpGlpK-KPN00353 complex (modeled structure) are listed in Table 2.

\section{Residue His65 of KPN00353 Is Important for Binding to GIpK and 1,3-PD Production}

To study the roles of residues His65 and His110 of KPN00353 in the interaction with GlpK, we generated His-tagged KPN00353 


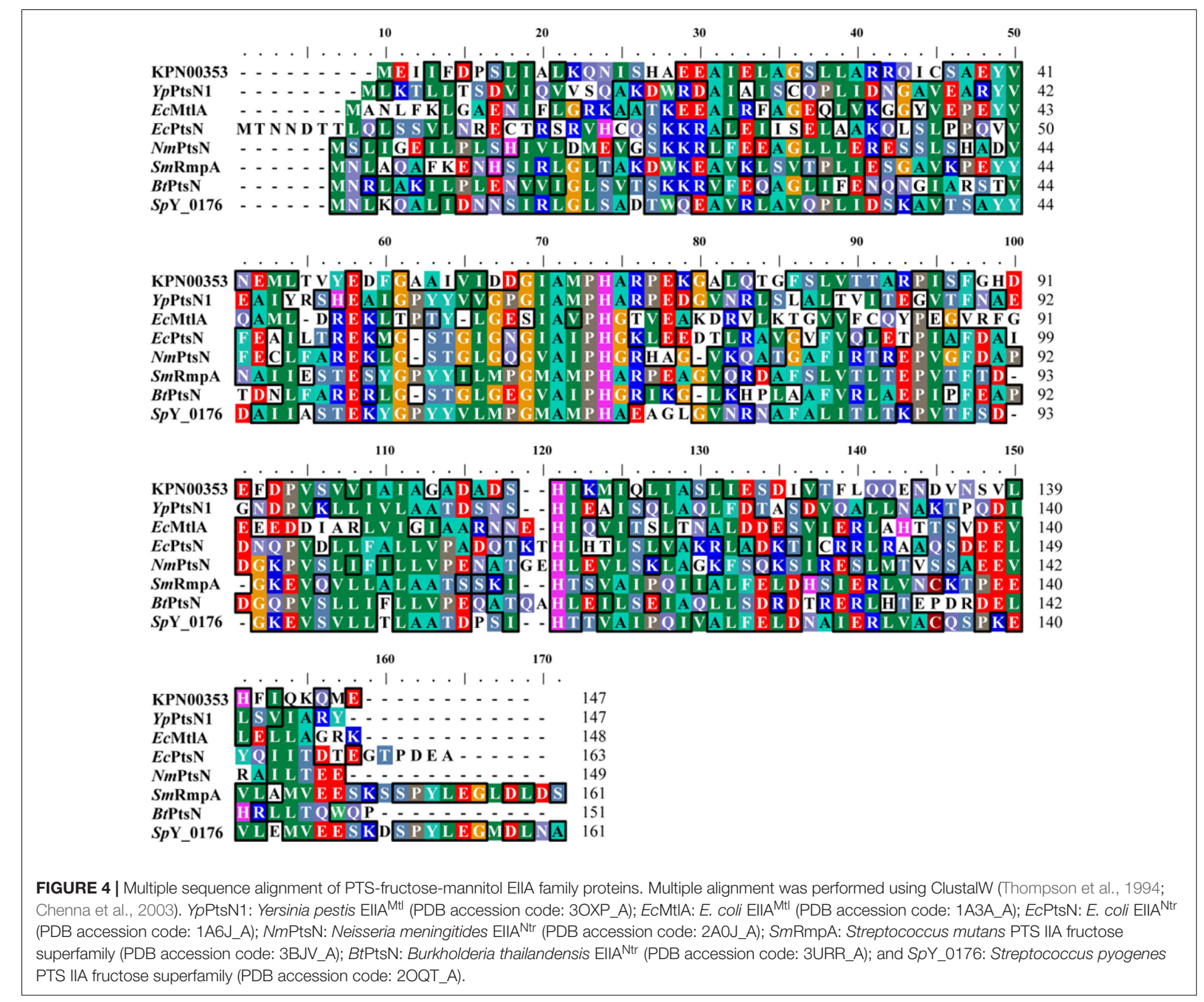

variants in which histidine was replaced with glutamine (H65Q and $\mathrm{H} 110 \mathrm{Q})$, aspartate $(\mathrm{H} 65 \mathrm{D})$, glutamate $(\mathrm{H} 65 \mathrm{E})$ or arginine (H65R). In the protein pull-down assay, GST-tagged GlpK bound to His-tagged H65D or His-tagged H65E mutant proteins more strongly than to His-tagged H65R or to His-tagged wild-type KPN00353 and bound weakly to His-tagged H65Q (Figure 8). The binding affinity between GlpK and the His-tagged H110Q mutant protein was similar to that between GlpK and His-tagged wild-type KPN00353 (Figure 8). To examine whether the binding affinity of KPN00353 variant and GlpK correlated with 1,3-PD production, we constructed plasmids containing the mutated KPN00353 genes under the control of the pBAD promoter and transformed them into K. pneumoniae MGH 78578 (Table 1). Then, we quantified the 1,3-PD from the recombinant $K$. pneumoniae overexpressing the KPN00353 variant. Compared with the vector control, all strains overexpressing the KPN00353 wild-type or variant protein exhibited reduced 1,3-PD production (Figure 8). Among them,
K. pneumoniae overexpressing the H65D, H65E or H65R variant produced lower levels of 1,3-PD than $K$. pneumoniae overexpressing the wild-type KPN00353. The 1,3-PD production of the strain overexpressing the H65Q variant was higher than that of K. pneumoniae overexpressing wild-type KPN00353 (Figure 8). The strain with the overexpressed H110Q variant produced a similar amount of 1,3-PD to that of the strain overexpressing wild-type KPN00353 (Figure 8). Furthermore, observation of the residual glycerol in the medium growing these bacteria showed an inverse relationship with 1,3-PD production: the lower the 1,3-PD production, the higher was the residual glycerol in the medium (Figure 8). Moreover, to examine the effect of the overexpression of different KPN00353 variants on the activity of GlpK, we quantified the intracellular G3P in recombinant K. pneumoniae overexpressing wild-type or variant KPN00353 proteins. The results of the G3P colorimetric assay showed that the amount of G3P was reduced in the strains overexpressing wild-type or variant KPN00353 


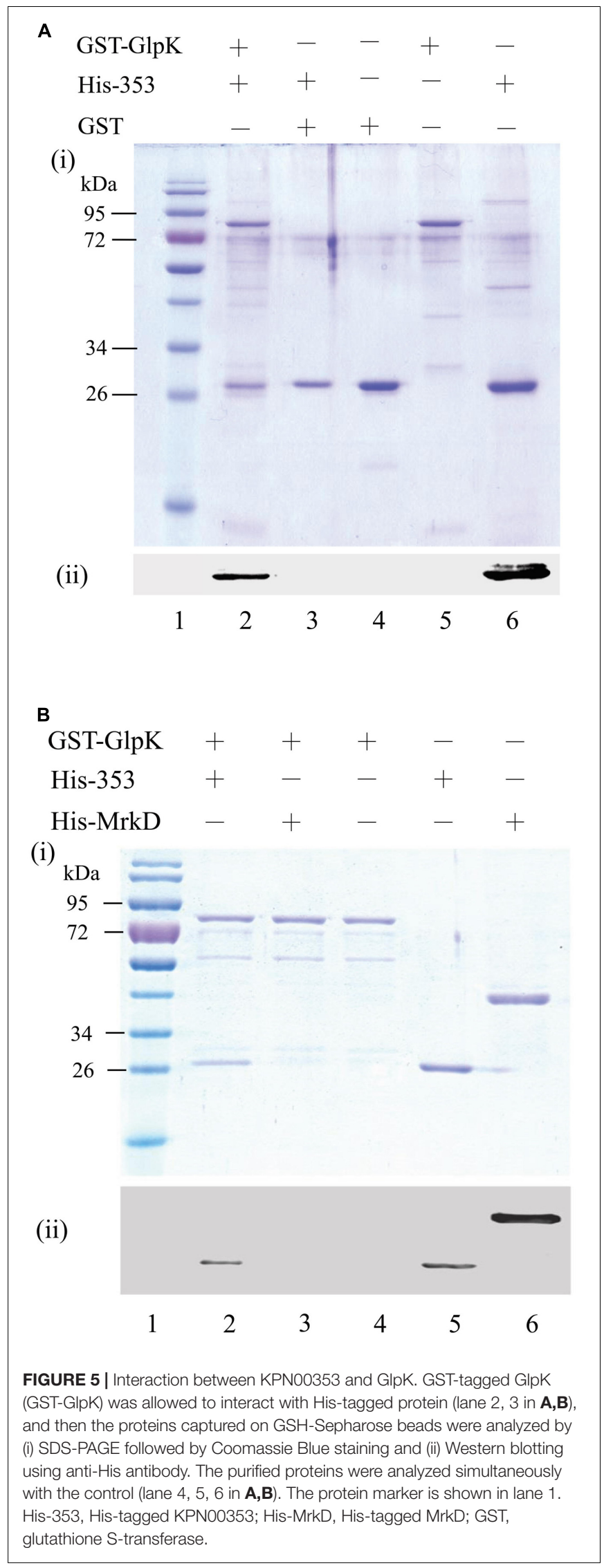

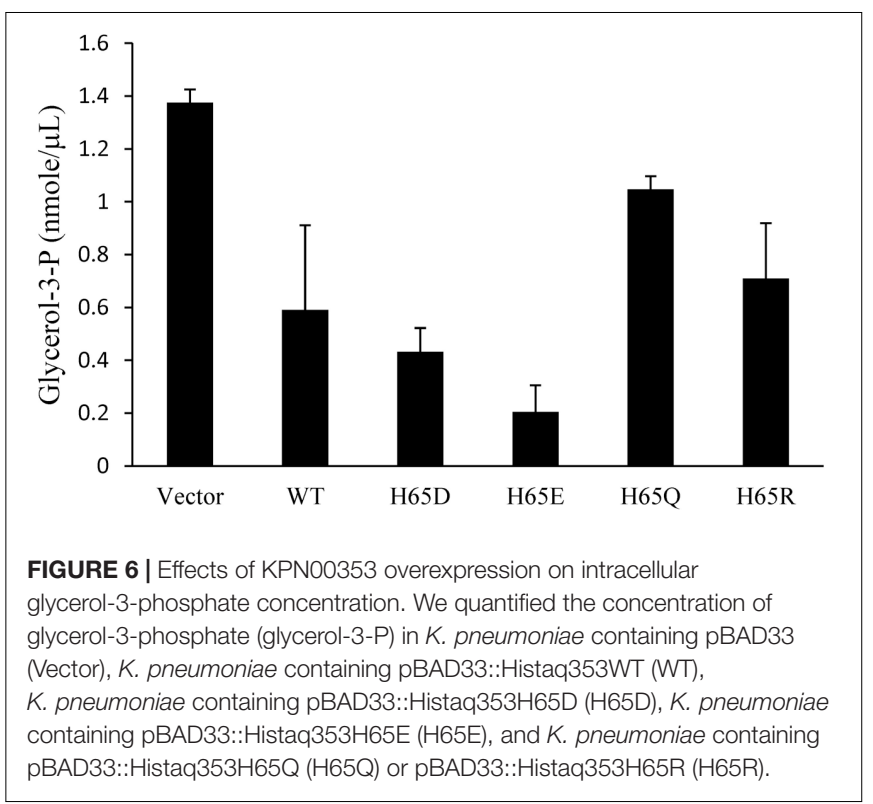

proteins compared with that in the vector control (Figure 6). Among them, K. pneumoniae overexpressing the $\mathrm{H} 65 \mathrm{E}$ variant produced the lowest level of G3P, while $K$. pneumoniae overexpressing $\mathrm{H} 65 \mathrm{Q}$ produced a lesser reduction in G3P level than $K$. pneumoniae overexpressing wild-type KPN00353 (Figure 6). We did not test the G3P in $K$. pneumoniae overexpressing $\mathrm{H} 110 \mathrm{Q}$ variant because the binding affinity of GlpK and the H110Q variant and the 1,3-PD production of recombinant $K$. pneumoniae overexpressing the $\mathrm{H} 110 \mathrm{Q}$ variant were not significantly different from the corresponding values for the wild-type KPN00353 (Figure 8). Therefore, these results indicated that the overexpression of KPN00353 inhibited the function of GlpK by binding to GlpK, leading to decreased 1,3-PD production.

\section{Structure Modeling and Comparison of Wild-Type and Mutant KPN00353}

To better understand the effects of the KPN00353 mutants on the binding affinity of KpGlpK-KPN00353 complex formation, structural models of $K p \mathrm{GlpK}$ complexed with the KPN00353 mutants were generated to interpret the contributions of the His65 and His110 residues of KPN00353 to the interface of the KpGlpK-KNP00353 complex. Residue His65 of KPN00353 may form a salt bridge with residue Asp326 of KpGlpK at physiological $\mathrm{pH}$ (Figure 9A). Moreover, residues His65 and His110 of KPN00353 can both contribute hydrophobic interactions with residue Phe328 of KpGlpK (Figure 9A). The H65E mutant of KPN00353 may form a hydrogen bond with residue Asp326 of $K p$ GlpK and a salt bridge with residue His110 of KPN00353 at physiological pH (Figure 9B). The H65D mutant of KPN00353 may form hydrogen bonds with residues Ser325 and Asp326 of KpGlpK and the main chain of residue Met63 of KPN00353 at physiological pH (Figure 9C). The H65R mutant of KPN00353 may form a salt bridge with residue Asp326 of KpGlpK and a hydrogen bond with the main chain of residue Gly104 of 

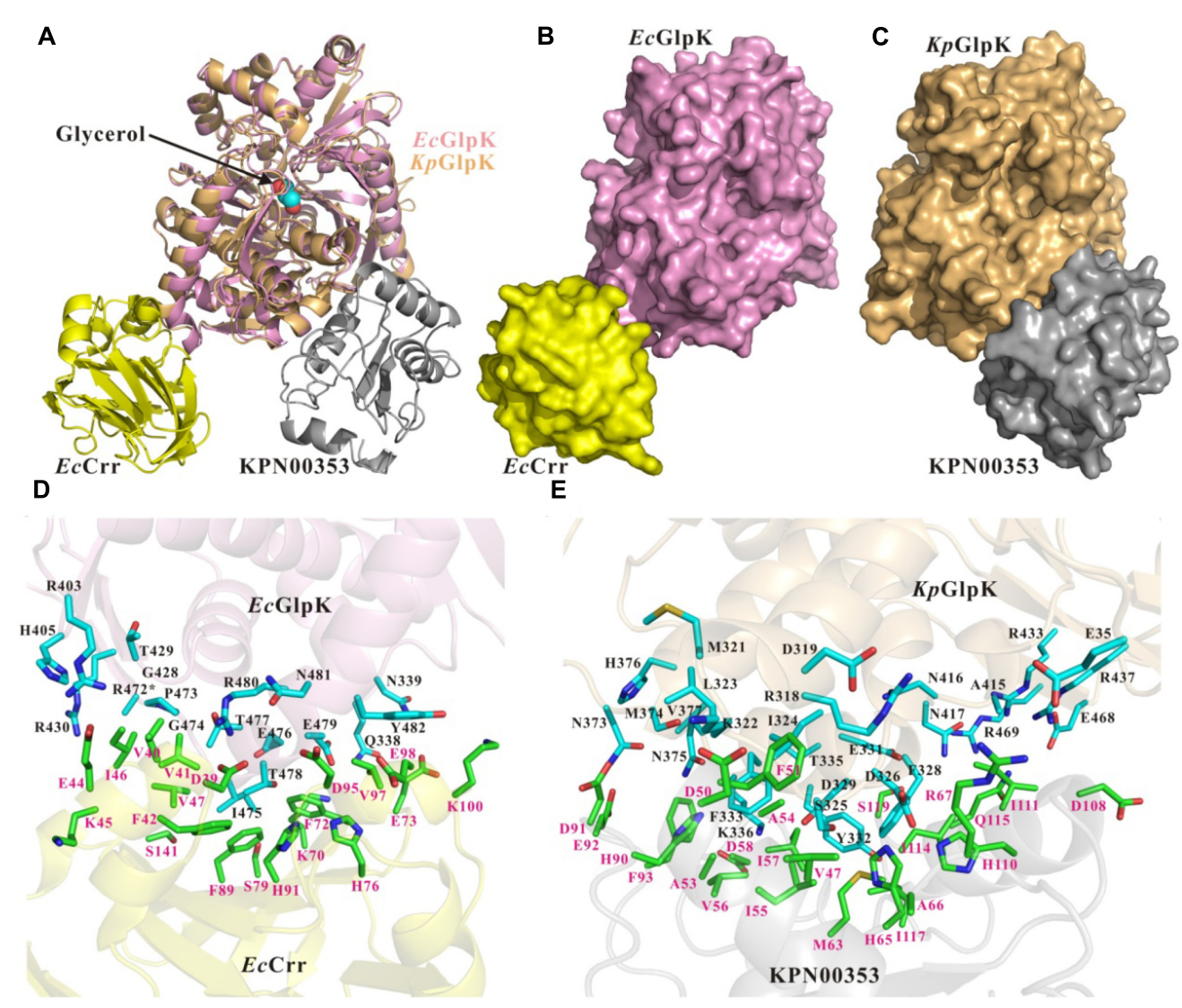

FIGURE 7| Structures and interface residues of the E. coli GlpK-Crr complex and K. pneumoniae GlpK-KPN00353 complex. (A) Superposition of cartoon representations of $E$. coli GlpK-Crr complex (solved structure, PDB accession code 1GLA) and K. pneumoniae GlpK-KPN00353 complex (modeled structure). Surface representations of (B) the E. coli GlpK-Crr complex and (C) the K. pneumoniae GlpK-KPN00353 complex in the same orientation as in (A). (D,E) Close-up view of the interfaces of the E. coli GlpK-Crr complex and the K. pneumoniae GlpK-KPN00353 complex, respectively. The E. coli GlpK, E. coli Crr, K. pneumoniae GlpK and KPN00353 molecules are shown in pink, yellow, light-orange and gray, respectively. The side chains of the interface residues of GlpK molecules are shown in cyan (carbon atoms) stick models and labeled in black. The side chains of the interface residues of the Crr and KPNO0353 molecules are shown in green (carbon atoms) stick models and labeled in magenta. The glycerol molecule is shown in a spheres model. Oxygen atoms are shown in red and nitrogen atoms in blue. ${ }^{*}$ The R472 side-chain atoms of E. coli GlpK are missing atoms in the 1GLA coordination file.

KPN00353 at physiological pH (Figure 9D). The H65Q mutant of KPN00353 may form a hydrogen bond with residue Asp326 of KpGlpK at physiological pH (Figure 9E). The H65E, H65D, H65R, H65Q and H110Q mutants of KPN00353 all reduce the hydrophobic interactions with residue Phe328 of KpGlpK (Figure 9).

\section{DISCUSSION}

In this study, we report that the overexpression of KPN00353, an EIIA homologue in K. pneumoniae, reduced both 1,3-PD production and glycerol uptake under microaerobic conditions via directly inhibiting the activity of GlpK. The KPN00353 homologue can be found in most K. pneumoniae isolates (Figure 1). Therefore, our findings regarding KPN00353 in K. pneumoniae MGH78578 can be applied to other K. pneumoniae isolates. However, we do not know what kind of environmental signal led to the inhibition of GlpK by KPN00353 in K. pneumoniae in this study. In E. coli and S. enterica serovar Typhimurium, environmental glucose decreases the extent of phosphorylation of EIIA ${ }^{\text {Glc }}$, while unphosphorylated EIIA ${ }^{\text {Glc }}$ binds efficiently to GlpK and inhibits the activity of GlpK in the presence of both glycerol and glucose (Postma et al., 1984; Deutscher et al., 2006). The overexpression of EIIA ${ }^{\text {Glc }}$ tends to be in the unphosphorylated form (Eppler et al., 2002). Therefore, in this study, we suppose that most overexpressed KPN00353 is unphosphorylated type and apt to bind with GlpK. Because we have not found the cognate sugar of KPN00353, overexpression of KPN00353 in medium containing glycerol without sugar may simulate the unphosphorylated state of KPN00353 in medium containing glycerol with the cognate sugar under microaerobic conditions. We presumed that the cognate sugar of KPN00353 has priority over glycerol for catabolism in K. pneumoniae under microaerobic conditions, leading reduced 1,3-PD production when the cognate sugar of KPN00353 and glycerol are both present in the medium. 
The 1,3-PD production of the $K$. pneumoniae glpK mutant and of the parent strain under anaerobic conditions did not differ significantly (Ashok et al., 2013). However, in our study, GlpK inhibition was correlated with reductions in G3P synthesis, glycerol uptake and 1,3-PD production in $K$. pneumoniae under microaerobic conditions (Figures 3, 6). Because of low $\mathrm{K}_{\mathrm{M}}$ of glycerol kinase toward glycerol, in the aerobic conditions, the major fraction of glycerol flows may through the respiratory route which GlpK is involved in (Kumar and Park, 2017). In addition, Voegele et al. (1993) reported that mutations in GlpK decreased glycerol transport in E. coli under aerobic conditions. We also observed the reduction of ethanol, one metabolite of oxidative pathway of glycerol metabolism, in K. pneumoniae overexpressing KPN00353, compared to vector control (data not shown). Therefore, we hypothesize that inhibition of GlpK by KPN00353 leads to decrease in glycerol

TABLE 2 | The interface residues of the E. coli GlpK-Crr complex (solved structure, PDB accession code 1GLA) and the K. pneumoniae GlpK-KPN00353 complex (modeled structure).

\begin{tabular}{|c|c|c|c|}
\hline \multicolumn{2}{|c|}{$\begin{array}{l}\text { Interface residues of } \\
\text { EcGlpK-Crr complex } \\
\text { (PDB accession code 1GLA) }\end{array}$} & \multicolumn{2}{|c|}{$\begin{array}{l}\text { Interface residues of } \\
\text { KpGlpK-KPN00353 complex } \\
\text { (modeled structure) }\end{array}$} \\
\hline EcGlpK & $E c \mathrm{Crr}$ & KpGlpK & KPN00353 \\
\hline Q338 & D39 & E35 & V47 \\
\hline N339 & V40 & R318 & D50 \\
\hline R403 & V41 & D319 & F51 \\
\hline $\mathrm{H} 405$ & F42 & M321 & G52 \\
\hline G428 & E44 & K322 & A53 \\
\hline T429 & K45 & L323 & A54 \\
\hline R430 & 146 & 1324 & 155 \\
\hline R472 & V47 & S325 & V56 \\
\hline P473 & K70 & D326 & 157 \\
\hline G474 & F72 & F328 & D58 \\
\hline 1475 & E73 & D329 & M63 \\
\hline E476 & $\underline{\mathrm{H}}^{\mathrm{b}} \underline{\mathrm{b}}^{\mathrm{b}}$ & E331 & $H 65^{a}$ \\
\hline T477 & S79 & Y332 & A66 \\
\hline T478 & F89 & F333 & R67 \\
\hline E479 & $\mathrm{H} 91^{\mathrm{a}}$ & T335 & H9O \\
\hline R480 & D95 & K336 & D91 \\
\hline N481 & V97 & V337 & E92 \\
\hline \multirow[t]{11}{*}{ Y482 } & E98 & N373 & F93 \\
\hline & K100 & S374 & D108 \\
\hline & $\mathrm{S} 141$ & N375 & ${\underline{\mathrm{H} 110^{\mathrm{b}}}}^{\mathrm{B}}$ \\
\hline & & H376 & 1111 \\
\hline & & A415 & 1114 \\
\hline & & N416 & Q115 \\
\hline & & N417 & 1117 \\
\hline & & R433 & A118 \\
\hline & & R437 & S119 \\
\hline & & E468 & \\
\hline & & R469 & \\
\hline
\end{tabular}

The interface residues were determined by PDBePISA, a website providing an interactive tool for the exploration of macromolecular interfaces. aThe histidine residue receiving the phosphoryl group from the donor HPr is indicated in bold. b The histidine residue closing the phosphorylation site is underlined.

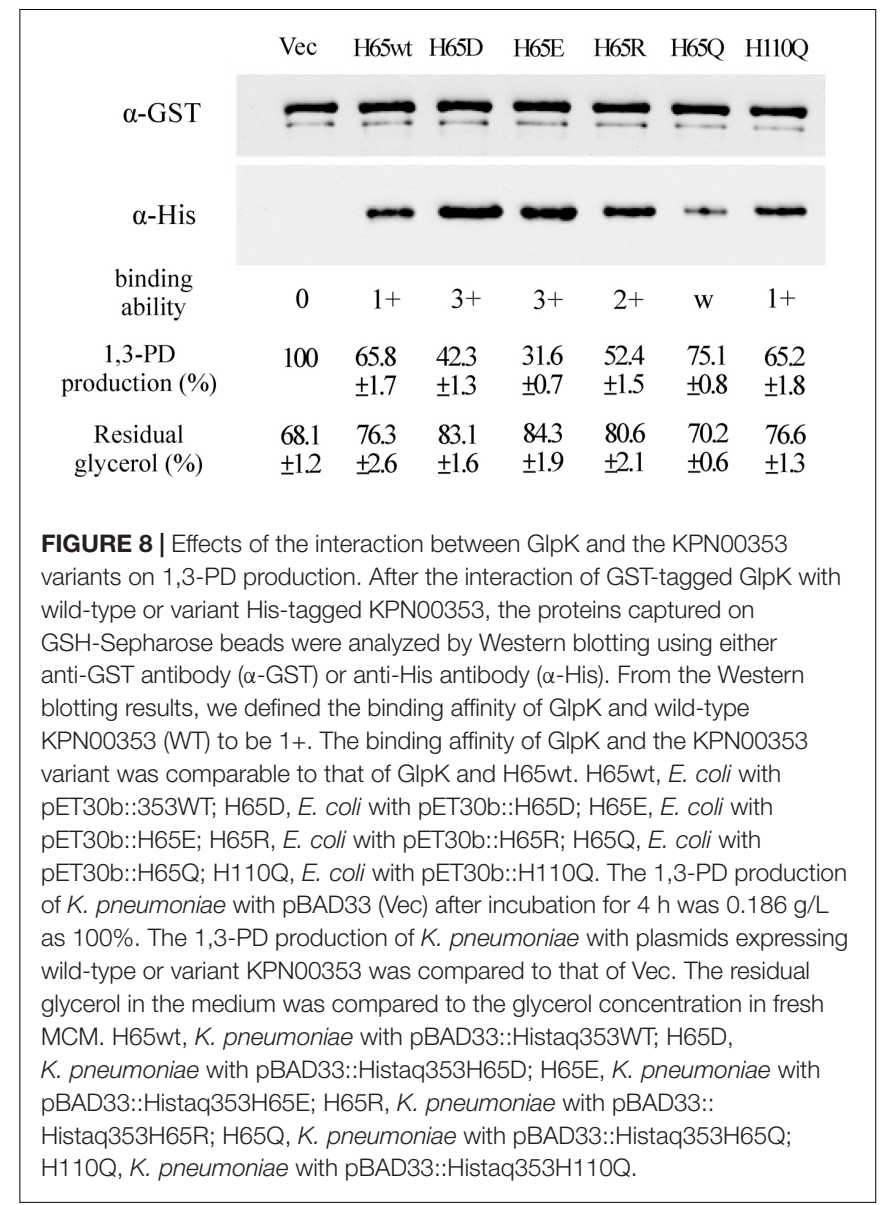

uptake under bacterial (micro)aerobic growth by unknown feedback mechanism. The decreased intracellular glycerol results in low activities of both oxidative and reductive pathways of glycerol metabolism in K. pneumoniae (Figure 10). In addition, Ashok reported that $g l p K$ mutant and wild-type K. pneumoniae demonstrated similar glycerol consumption and 1,3-PD production profiles under anaerobic condition with addition of nitrate as electron acceptor. The authors suggested the involvement of other kinases in glycerol metabolism (Ashok et al., 2013; Kumar and Park, 2017). Therefore, we suggest that overexpression of KPN00353 inhibits the functions of GlpK and other kinases playing similar roles in glycerol metabolism. Because 1,3-PD production by K. pneumoniae under microaerobic conditions has several benefits (Chen X. et al., 2003), the effects of the EIIA homolog or its cognate sugar on GlpK and 1,3-PD production under microaerobic conditions should be evaluated.

The gene $\mathrm{crr}$ encodes glucose-specific EIIA in E. coli and S. enterica serovar Typhimurium. Oh et al. (2013) reported that the production of 1,3-PD by the K. pneumoniae crr mutant strain in the presence of both glucose and glycerol in the medium is enhanced compared with the production of 1,3-PD by the parent strain. However, the production of 1,3-PD by the crr mutant and the parent strain in the medium containing glycerol without glucose was similar to the $1,3-\mathrm{PD}$ production 

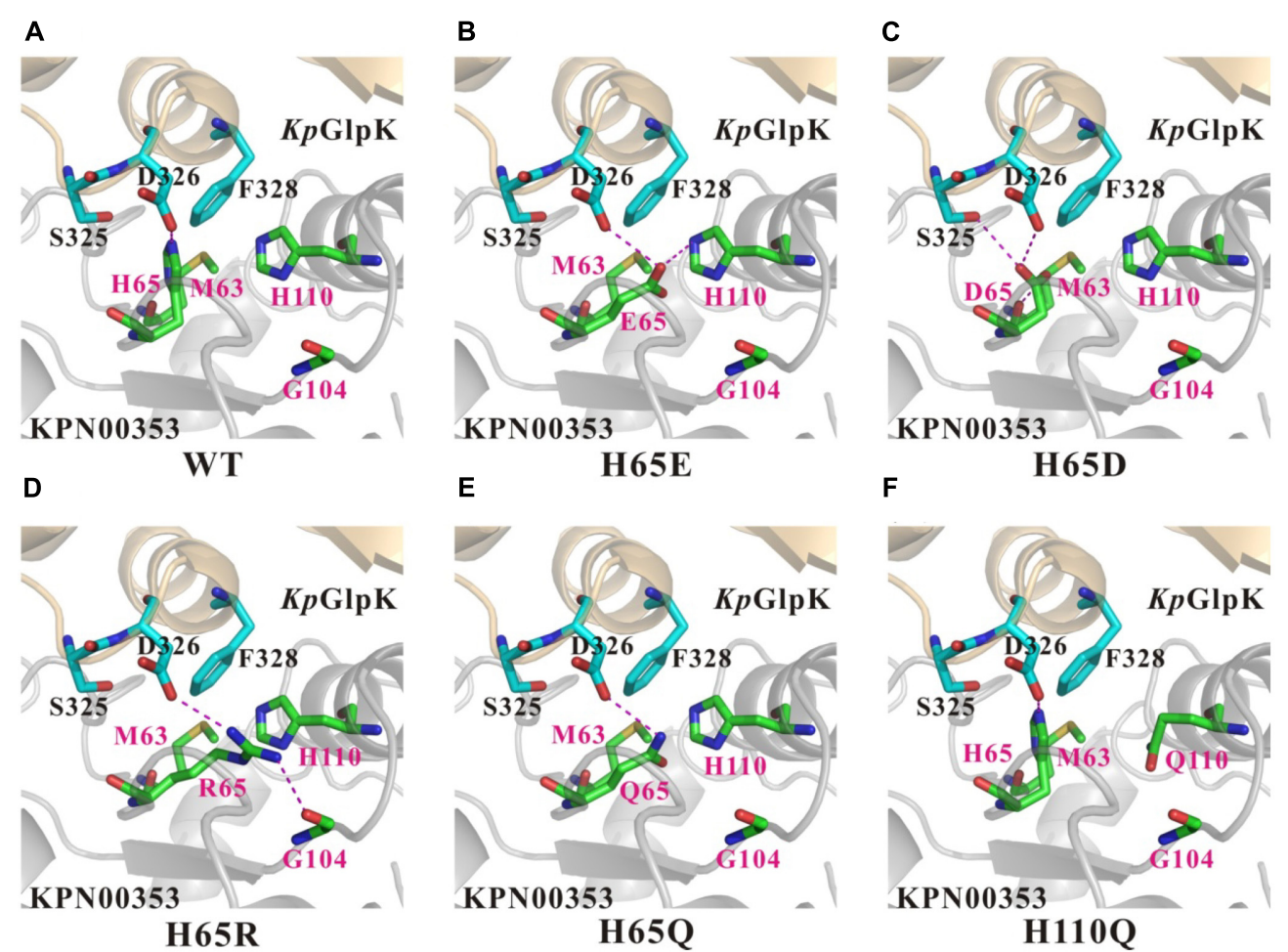

$\mathbf{F}$

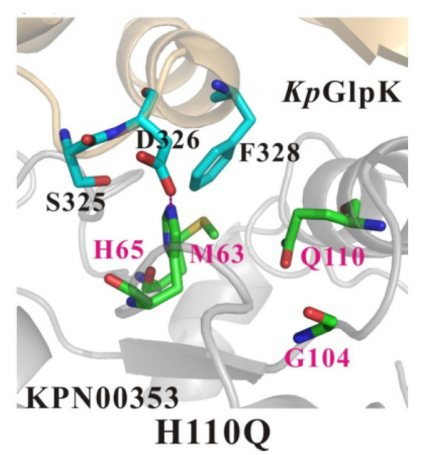

FIGURE 9 | Comparison of the contacts surrounding residues 65 and 110 of KPN00353 variants interacting with K. pneumoniae GlpK. (A) Wild-type KPN00353, (B) KPN00353 H65E mutant, (C) KPN00353 H65D mutant, (D) KPN00353 H65R mutant, (E) KPN00353 H65Q mutant, and (F) KPN00353 H110Q mutant interacting with K. pneumoniae GlpK. The main chain and side chains of the interface residues of the GlpK molecules are shown in cyan (carbon atoms) stick models and labeled in black. The main chains and side chains of the interface residues of the KPN00353 molecules are shown in green (carbon atoms) stick models and labeled in magenta. The critical salt bridges or hydrogen bonds are indicated by magenta dashed lines.
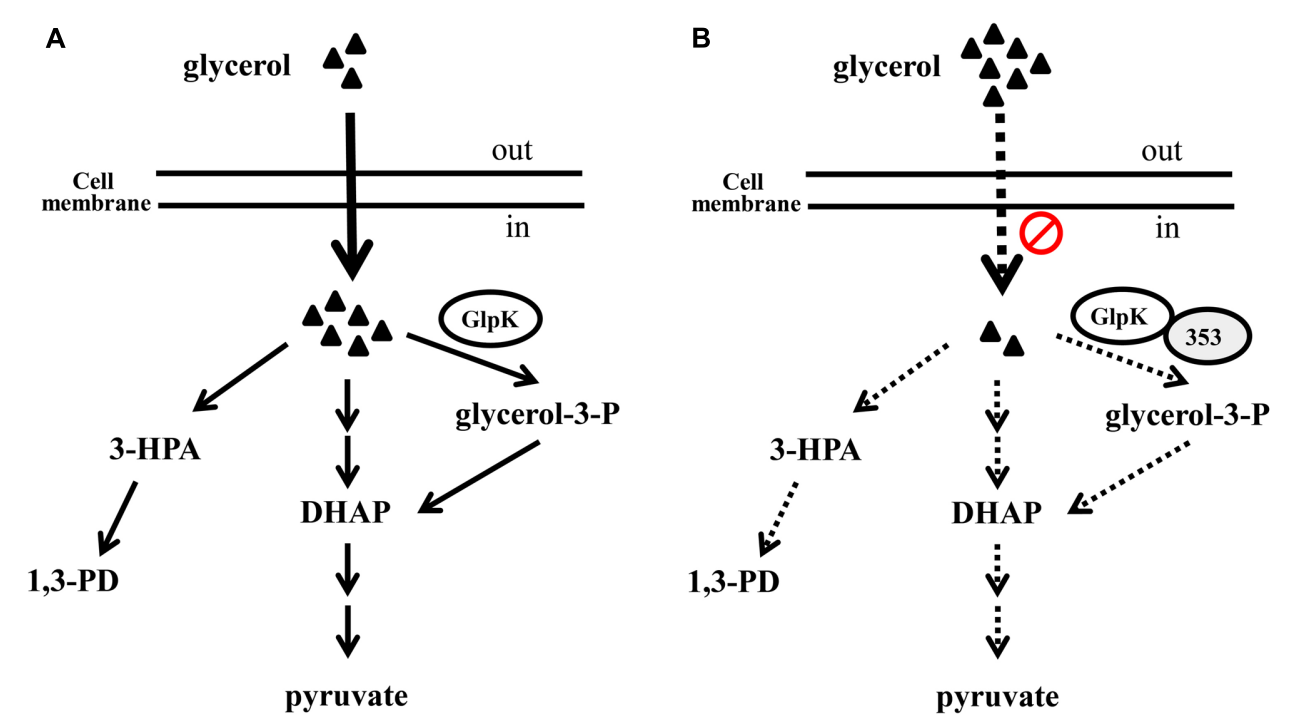

FIGURE 10 | The hypothetical role of KPN00353 in glycerol metabolism in K. pneumoniae. Glycerol is metabolized by respiratory metabolism which GlpK is involved in and oxidative fermentation to produced DHAP or by reductive pathway to produce 1,3-PD under (micro)aerobic condition (A). We hypothesize that the interaction of GlpK and KPN00353 (353) decreases the glycerol uptake into bacterium (in B). The reduction of intracellular glycerol results in the low activity of glycerol metabolism. Dash lines in (B) indicate the activities of reactions or pathways in (B) are lower than those represented by solid lines in (A). Triangle indicates glycerol. 
by the $\mathrm{crr}$ mutant strain in the presence of both glucose and glycerol (Oh et al., 2013). Many studies have developed various biotechnological processes, including process and genetic engineering approaches, to improve the production of 1,3-PD by microorganisms. Genetic engineering approaches include the overexpression of homologous or heterologous genes involved in the 1,3-PD synthesis pathway (Ma et al., 2010) and the deletion of genes involved in byproduct formation (Horng et al., 2010). Sen et al. (2015) reported that the addition of glucose to the glycerol fermentation led to increased cell mass but no improvement in the 1,3-PD production of K. pneumoniae. There are several EIIA homologs belonging to different families in K. pneumoniae. ${ }^{2}$ Our findings and the report by Oh et al. show that the deletion of EIIA does not enhance the 1,3 PD production of $K$. pneumoniae in medium containing glycerol without the cognate sugar (Figure 3) (Oh et al., 2013). However, we surmise that the KPN00353 mutant would retain a high level of 1,3-PD production in medium containing glycerol with the cognate sugar, such as the crr mutant in the medium containing glycerol with glucose (Oh et al., 2013).

The typical energies of salt bridge, hydrogen bond, and hydrophobic interactions are approximately $2 \mathrm{kcal} / \mathrm{mol}, 1$ $\mathrm{kcal} / \mathrm{mol}$, and $0.7 \mathrm{kcal} / \mathrm{mol}$, respectively. The $\mathrm{pKa}$ values of aspartate $(\mathrm{D})$, glutamate $(\mathrm{E})$, histidine $(\mathrm{H})$ and arginine $(\mathrm{R})$ side chains in unfolded protein are 3.9, 4.1, 6.0 and 12.5, respectively. However, the $\mathrm{pKa}$ values of aspartate and glutamate decrease in a folded protein, whereas the $\mathrm{pKa}$ values of histidine and arginine increase. Therefore, glutamate and aspartate are negatively charged residues, whereas arginine is a positively charged residue and histidine is a partially positively charged residue under the conditions of a protein pull-down assay. In contrast, with glutamate, aspartate and arginine, histidine provides weak affinity via salt-bridge and hydrogen-bond formation. As mentioned above, H65D, H65E and H65R KPN00353 mutants have stronger binding affinities with KpGlpK than wild-type KPN00353. On the other hand, glutamine (Q) has an uncharged side chain, and therefore, H65Q has a weaker binding affinity with $K p$ GlpK than does wild-type KPN00353. Moreover, the H110Q KPN00353 mutant provides similar binding affinity to that of wild-type KPN00353 because residues H110 and Q110 provide hydrophobic interactions only with KpGlpK.

${ }^{2}$ http://www.genome.jp/kegg/kegg2.html

\section{REFERENCES}

Al Makishah, N. H., and Mitchell, W. J. (2013). Dual substrate specificity of an $\mathrm{N}$-acetylglucosamine phosphotransferase system in Clostridium beijerinckii. Appl. Environ. Microbiol. 79, 6712-6718. doi: 10.1128/AEM. 01866-13

Ashok, S., Mohan Raj, S., Ko, Y., Sankaranarayanan, M., Zhou, S., Kumar, V., et al. (2013). Effect of puuC overexpression and nitrate addition on glycerol metabolism and anaerobic 3-hydroxypropionic acid production in recombinant Klebsiella pneumoniae DeltaglpKDeltadhaT. Metab. Eng. 15, 10-24. doi: 10.1016/j.ymben.2012.09.004

Bagley, S. T. (1985). Habitat association of Klebsiella species. Infect. Control 6, 52-58. doi: 10.1017/s0195941700062603
Apart from the glucose family, there are as yet no reports of other EIIA homologues binding to GlpK. In this study, KPN00353, a member of the fructose-mannitol EIIA (EII ${ }^{\mathrm{Fru}}$ and EIIA $^{\mathrm{Mtl}}$ ) family, was observed to bind to GlpK (Figure 5). By structure modeling and the construction of KPN00353 variants, we identified the important residues of EIIA in the fructosemannitol EIIA family for protein binding and, hence, for the GlpK activity and 1,3-PD production (Figures 6, 7, 8).

\section{AUTHOR CONTRIBUTIONS}

W-YJ: performed the experiments, analyzed the data, wrote the manuscript and revised the work critically. NP: performed the experiments and analyzed the data. Y-TH: wrote the manuscript and revised the work critically. W-TC: performed the experiments and analyzed the data. C-CC: provide opinion. P-CS: conceived, designed and performed the experiments, analyzed the data, revised the work critically and procured funding.

\section{FUNDING}

This work was supported by a grant (MOST 103-2320-B320-008-MY3) from the Ministry of Science and Technology and a grant (TCIRP99002-04) from Tzu Chi University. This work is also partially supported by the Taiwan Protein Project (MOST105-0210-01-12-01 and MOST106-0210-01-15-04) and the Program for Translational Innovation of Biopharmaceutical Development - Technology Supporting Platform Axis.

\section{ACKNOWLEDGMENT}

The authors thank Mr. Chih-Chun Kuo for his contributions to this work.

\section{SUPPLEMENTARY MATERIAL}

The Supplementary Material for this article can be found online at: https://www.frontiersin.org/articles/10.3389/fmicb. 2017.02441/full\#supplementary-material

Bordo, D., van Monfort, R. L., Pijning, T., Kalk, K. H., Reizer, J., Saier, M. H., et al. (1998). The three-dimensional structure of the nitrogen regulatory protein IIANtr from Escherichia coli. J. Mol. Biol. 279, 245-255. doi: 10.1006/jmbi.1998. 1753

Celinska, E. (2012). Klebsiella spp as a 1,3-propanediol producer: the metabolic engineering approach. Crit. Rev. Biotechnol. 32, 274-288. doi: 10.3109/ 07388551.2011.616859

Chen, R., Li, L., and Weng, Z. (2003). ZDOCK: an initial-stage protein-docking algorithm. Proteins 52, 80-87. doi: 10.1002/prot.10389

Chen, X., Xiu, Z., Wang, J., Zhang, D., and Xu, P. (2003). Stoichiometric analysis and experimental investigation of glycerol bioconversion to 1,3-propanediol by Klebsiella pneumoniae under microaerobic conditions. Enzyme Microb. Technol. 33, 386-394. doi: 10.1016/S0141-0229(03)00135-2 
Chenna, R., Sugawara, H., Koike, T., Lopez, R., Gibson, T. J., Higgins, D. G., et al. (2003). Multiple sequence alignment with the clustal series of programs. Nucleic Acids Res. 31, 3497-3500. doi: 10.1093/nar/gkg500

Deutscher, J., Ake, F. M., Derkaoui, M., Zebre, A. C., Cao, T. N., Bouraoui, H., et al. (2014). The bacterial phosphoenolpyruvate:carbohydrate phosphotransferase system: regulation by protein phosphorylation and phosphorylation-dependent protein-protein interactions. Microbiol. Mol. Biol. Rev. 78, 231-256. doi: 10.1128/MMBR.00001-14

Deutscher, J., Francke, C., and Postma, P. W. (2006). How phosphotransferase system-related protein phosphorylation regulates carbohydrate metabolism in bacteria. Microbiol. Mol. Biol. Rev. 70, 939-1031. doi: 10.1128/MMBR.00024-06

Emsley, P., and Cowtan, K. (2004). Coot: model-building tools for molecular graphics. Acta Crystallogr. D Biol. Crystallogr. 60, 2126-2132. doi: 10.1107/ S0907444904019158

Eppler, T., Postma, P., Schutz, A., Volker, U., and Boos, W. (2002). Glycerol-3phosphate-induced catabolite repression in Escherichia coli. J. Bacteriol. 184, 3044-3052. doi: 10.1128/JB.184.11.3044-3052.2002

Forage, R. G., and Lin, E. C. (1982). DHA system mediating aerobic and anaerobic dissimilation of glycerol in Klebsiella pneumoniae NCIB 418. J. Bacteriol. 151, 591-599.

Guo, N. N., Zheng, Z. M., Mai, Y. L., Liu, H. J., and Liu, D. H. (2010). Consequences of mutation of Klebsiella pneumoniae on 1,3-propanediol fermentation. Appl. Microbiol. Biotechnol. 86, 701-707. doi: 10.1007/s00253-009-2342-8

Guzman, L. M., Belin, D., Carson, M. J., and Beckwith, J. (1995). Tight regulation, modulation, and high-level expression by vectors containing the arabinose PBAD promoter. J. Bacteriol. 177, 4121-4130.

Horng, Y. T., Chang, K. C., Chou, T. C., Yu, C. J., Chien, C. C., Wei, Y. H., et al. (2010). Inactivation of $d h a D$ and $d h a K$ abolishes by-product accumulation during 1,3-propanediol production in Klebsiella pneumoniae. J. Ind. Microbiol. Biotechnol. 37, 707-716. doi: 10.1007/s10295-010-0714-9

Horng, Y.-T., Wang, C.-J., Chung, W.-T., Chao, H.-J., Chen, Y.-Y., and Soo, P.-C. (2017). Phosphoenolpyruvate phosphotransferase system components positively regulate Klebsiella biofilm formation. J. Microbiol. Immunol. Infect. doi: 10.1016/j.jmii.2017.01.007 [Epub ahead of print]

Huang, H., Gong, C. S., and Tsao, G. T. (2002). Production of 1,3-propanediol by Klebsiella pneumoniae. Appl. Biochem. Biotechnol. 98-100, 687-698.

Hurley, J. H., Faber, H. R., Worthylake, D., Meadow, N. D., Roseman, S., Pettigrew, D. W., et al. (1993). Structure of the regulatory complex of Escherichia coli IIIGlc with glycerol kinase. Science 259, 673-677.

Krissinel, E., and Henrick, K. (2004). Secondary-structure matching (SSM), a new tool for fast protein structure alignment in three dimensions. Acta Crystallogr. D Biol. Crystallogr. 60, 2256-2268. doi: 10.1107/S0907444904026460

Krissinel, E., and Henrick, K. (2007). Inference of macromolecular assemblies from crystalline state. J. Mol. Biol. 372, 774-797. doi: 10.1016/j.jmb.2007.05.022

Kumar, V., and Park, S. (2017). Potential and limitations of Klebsiella pneumoniae as a microbial cell factory utilizing glycerol as the carbon source. Biotechnol. $A d v$. doi: 10.1016/j.biotechadv.2017.10.004 [Epub ahead of print]

Liang, Q., Zhang, F., Li, Y., Zhang, X., Li, J., Yang, P., et al. (2015). Comparison of individual component deletions in a glucose-specific phosphotransferase system revealed their different applications. Sci. Rep. 5:13200. doi: 10.1038/ srep 13200

Ma, Z., Rao, Z., Xu, L., Liao, X., Fang, H., Zhuge, B., et al. (2010). Expression of dha operon required for 1,3-PD formation in Escherichia coli and Saccharomyces cerevisiae. Curr. Microbiol. 60, 191-198. doi: 10.1007/s00284-009-9528-2

McClelland, M., Florea, L., Sanderson, K., Clifton, S. W., Parkhill, J., Churcher, C., et al. (2000). Comparison of the Escherichia coli K-12 genome with sampled genomes of a Klebsiella pneumoniae and three salmonella enterica serovars, Typhimurium, Typhi and Paratyphi. Nucleic Acids Res. 28, 4974-4986. doi: 10.1093/nar/28.24.4974

Medrano, E. G., Forray, M. M., and Bell, A. A. (2014). Complete genome sequence of a Klebsiella pneumoniae strain isolated from a known cotton insect boll vector. Genome Announc. 2:e00850-14 doi: 10.1128/genomeA.00850-14

Oh, B. R., Hong, W. K., Heo, S. Y., Luo, L. H., Kondo, A., Seo, J. W., et al. (2013). The production of 1,3-propanediol from mixtures of glycerol and glucose by a Klebsiella pneumoniae mutant deficient in carbon catabolite repression. Bioresour. Technol. 130, 719-724. doi: 10.1016/j.biortech.2012.12.076
Postma, P. W., Epstein, W., Schuitema, A. R., and Nelson, S. O. (1984). Interaction between IIIGlc of the phosphoenolpyruvate:sugar phosphotransferase system and glycerol kinase of Salmonella typhimurium. J. Bacteriol. 158, 351-353.

Pyne, M. E., Liu, X., Moo-Young, M., Chung, D. A., and Chou, C. P. (2016). Genome-directed analysis of prophage excision, host defence systems, and central fermentative metabolism in Clostridium pasteurianum. Sci. Rep. 6:26228. doi: 10.1038/srep26228

Sambrook, J., Fritsch, E. F., and Maniatis, T. (1989). Molecular Cloning: A Laboratory Manual. Cold Spring Harbor, NY: Cold Spring Harbor Laboratory Press.

Saxena, R. K., Anand, P., Saran, S., and Isar, J. (2009). Microbial production of 1,3-propanediol: recent developments and emerging opportunities. Biotechnol. Adv. 27, 895-913. doi: 10.1016/j.biotechadv.2009.07.003

Schurtz, T. A., Hornick, D. B., Korhonen, T. K., and Clegg, S. (1994). The type 3 fimbrial adhesin gene (mrkD) of Klebsiella species is not conserved among all fimbriate strains. Infect. Immun. 62, 4186-4191.

Sen, B., Dabir, A. P., Lanjekar, V. B., and Ranade, D. R. (2015). Isolation and partial characterization of a new strain of Klebsiella pneumoniae capable of high 1,3 propanediol production from glycerol. Glob. J. Environ. Sci. Manage. 1, 99-108. doi: 10.7508/gjesm.2015.02.001

Sliz, P., Engelmann, R., Hengstenberg, W., and Pai, E. F. (1997). The structure of enzyme IIA lactose from Lactococcus lactis reveals a new fold and points to possible interactions of a multicomponent system. Structure 5, 775-788.

Soding, J., Biegert, A., and Lupas, A. N. (2005). The HHpred interactive server for protein homology detection and structure prediction. Nucleic Acids Res. 33, W244-W248. doi: 10.1093/nar/gki408

Soo, P. C., Wei, J. R., Horng, Y. T., Hsieh, S. C., Ho, S. W., and Lai, H. C. (2005). Characterization of the dapA-nlpB genetic locus involved in regulation of swarming motility, cell envelope architecture, hemolysin production, and cell attachment ability in Serratia marcescens. Infect. Immun. 73, 6075-6084. doi: 10.1128/IAI.73.9.6075-6084.2005

Sullivan, C. J. (1993). “Propanediols," in Ullmann's Encyclopedia of Industrial Chemistry, eds B. Elvers, S. Hawkins, W. Russey, and G. Schulz (New York, NY: VCH Publishers), 163-171.

Thompson, J. D., Higgins, D. G., and Gibson, T. J. (1994). Clustal-wimproving the sensitivity of progressive multiple sequence alignment through sequence weighting, position-specific gap penalties and weight matrix choice. Nucleic Acids Res. 22, 4673-4680. doi: 10.1093/nar/22.22.4673

Voegele, R. T., Sweet, G. D., and Boos, W. (1993). Glycerol kinase of Escherichia coli is activated by interaction with the glycerol facilitator. J. Bacteriol. 175, 1087-1094.

Wang, Z. X., Zhuge, J., Fang, H., and Prior, B. A. (2001). Glycerol production by microbial fermentation: a review. Biotechnol. Adv. 19, 201-223.

Wehtje, C., Beijer, L., Nilsson, R. P., and Rutberg, B. (1995). Mutations in the glycerol kinase gene restore the ability of a ptsGHI mutant of Bacillus subtilis to grow on glycerol. Microbiology 141, 1193-1198. doi: 10.1099/13500872-1415-1193

Wu, M. C., Chen, Y. C., Lin, T. L., Hsieh, P. F., and Wang, J. T. (2012). Cellobiosespecific phosphotransferase system of Klebsiella pneumoniae and its importance in biofilm formation and virulence. Infect. Immun. 80, 2464-2472. doi: 10.1128/ IAI.06247-11

Zeng, A. P., and Biebl, H. (2002). Bulk chemicals from biotechnology: the case of 1,3-propanediol production and the new trends. Adv. Biochem. Eng. Biotechnol. 74, 239-259. doi: 10.1007/3-540-45736-4_11

Conflict of Interest Statement: The authors declare that the research was conducted in the absence of any commercial or financial relationships that could be construed as a potential conflict of interest.

Copyright (c) 2017 Jeng, Panjaitan, Horng, Chung, Chien and Soo. This is an openaccess article distributed under the terms of the Creative Commons Attribution License (CC BY). The use, distribution or reproduction in other forums is permitted, provided the original author(s) or licensor are credited and that the original publication in this journal is cited, in accordance with accepted academic practice. No use, distribution or reproduction is permitted which does not comply with these terms. 